\title{
On a new method for the stochastic perturbation of the disease transmission coefficient in SIS Models
}

\author{
Student: Berk Tan Perçin ${ }^{1}$ \\ Supervisor: Alberto Lanconelli2 ${ }^{2}$
}

September 20, 2021

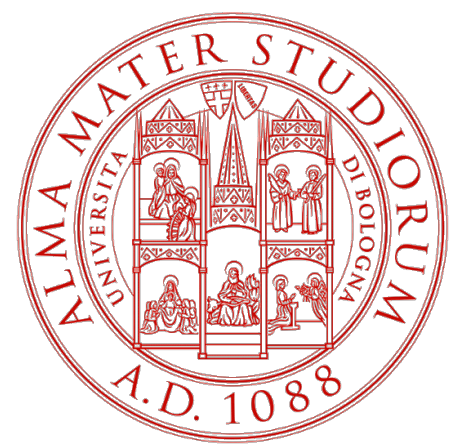

ALMA MATER STUDIORUM
UNIVERSITÂ DI BOLOGNA

\footnotetext{
${ }^{1}$ Dipartimento di Fisica e Astronomia Augusto Righi, Università di Bologna, Bologna, Italy. e-mail: berktan.percin@studio.unibo.it

${ }^{2}$ Dipartimento di Scienze Statistiche Paolo Fortunati, Universitá di Bologna, Bologna, Italy. e-mail: alberto.lanconelli2@unibo.it
} 


\begin{abstract}
In this study, first a necessary background is provided to readers. Then a novel approach to stochastically perturb the disease transmission coefficient was investigated, which is a key parameter in susceptible-infected-susceptible (SIS) models. Motivated by the papers [5] and [2], the disease transmission coefficient was perturbed with a Gaussian white noise, formally modelled as the time derivative of a mean reverting Ornstein-Uhlenbeck process. It has been remarked that, thanks to a suitable representation of the solution to the deterministic SIS model, this perturbation is rigorous and supported by a WongZakai approximation argument that consists in smoothing the singular Gaussian white noise and then taking limit of the solution from the approximated model. It has been proven that the stochastic version of the classic SIS model obtained this way preserves a crucial feature of the deterministic equation: the reproduction number dictating the two possible asymptotic regimes for the infection, i.e. extinction and persistence, remains unchanged. Then the class of perturbing noises for which this property holds were identified and propose simple sufficient conditions for that. All the theoretical discoveries are illustrated and discussed with the help of several numerical simulations.
\end{abstract}




\section{Contents}

\begin{tabular}{lll}
\hline & Introduction & 2
\end{tabular}

1.1 Background . . . . . . . . . . . . . . . . . . . . . 2

1.1.1 The Basics Of Probability Theory . . . . . . . . . . . . . . . 2

1.1 .2 Stochastic Processes . . . . . . . . . . . . . . . . . . 3

1.1 .3 Brownian Motion . . . . . . . . . . . . . . . . . . . . 4

1.1.4 Filtrations, Martingales and Conditional Expectation . . . . . . . 5

1.1.5 The Itô Integral . . . . . . . . . . . . . . . . . . . . . . . 7

$1.1 .6 \quad$ Stochastic Differential Equations (SDEs) . . . . . . . . . . . . . . . . . . 9

1.1.7 Wong-Zakai Theorem . . . . . . . . . . . . . . . . . . . . . . . . . 13

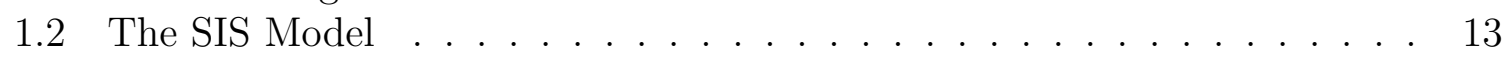

1.3 Perturbing The SIS Model In Literature . . . . . . . . . . . . . . . . 15

$\begin{array}{lll}2 & \text { The Analysis Performed In The Thesis } & 17\end{array}$

$2.1 \quad$ Stochastic parameter perturbation with a mean reverting Ornstein-Uhlenbeck

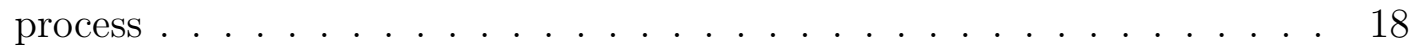

2.1.1 Cross-validation of the model via Wong-Zakai theorem . . . . . . 19

$2.2 \quad$ Analysis of the stochastically perturbed SIS Model . . . . . . . . . . . 20

2.2 .1 Support of the solution . . . . . . . . . . . . . . . . . . 20

$2.2 .2 \quad$ Extinction of the infection . . . . . . . . . . . . . . . . . . . . 21

2.2 .3 Persistence of the Infection . . . . . . . . . . . . . . . . . . . 22

2.2 .4 Trajectory simulations . . . . . . . . . . . . . . . . 24

$\begin{array}{|ll|}3 & \text { Generalization of The Parameter Perturbation Process } \\ 27\end{array}$

3.1 Stochastic parameter perturbation with a general process . . . . . . . . . 27

3.2 Trajectory simulations $\ldots \ldots \ldots \ldots \ldots \ldots \ldots$

$\begin{array}{|ll|}4 \text { Conclusion and Discussion } & 34\end{array}$ 


\section{Chapter 1}

\section{Introduction}

\subsection{Background}

Before the start of the analysis, first the necessary background will be provided for readers to fully grasp the topic. Later, the the research going on in literature will be introduced, so that the readers will know what has been done and what improvements can be done in the field. Lastly the research that has been conducted will be provided. It should be mentioned that the main parts of this work is already published in [17] and this document will re-state them.

\subsubsection{The Basics Of Probability Theory}

Probability Theory is the most crucial tool to build a framework in stochastic processes. This is why, in this study the basics of Probability Theory will be stated to make it easier for the reader.

\section{The $\sigma$-algebra}

The $\sigma$-algebra is a necessary element to construct the probability space that, one is going to work in. Moreover, when working with stochastic processes, the terms being adapted or being independent is also linked with the $\sigma$-algebras. Hence this section is dedicated for a brief introduction of the object and a more in depth reading can be done on the book [18].

The $\sigma$-algebra or $\sigma$-field ( of $\Omega$ ) is, by the definition on [18], a set that is constructed by countable addition, intersection and complementation of subsets of $\Omega$. A $\sigma$-algebra $\mathcal{F}$ have to satisfy the following:

- $\Omega \in \mathcal{F}$.

- $A \in \mathcal{F}$ implies $A^{c} \in \mathcal{F}$.

- $A_{i} \in \mathcal{F}$ for $i \geq 1$ implies that $\cup_{i=1}^{\infty} A_{i} \in \mathcal{F}$. 
As one can see, what a $\sigma$-algebra provides is, a closed space under basic set operations such as union, intersection and complementation. This is useful in probability theory because, even though one can perform these operations on some events (subsets of sample space $\Omega$ ), he/she will never be working outside of the $\sigma$-algebra.

\section{limsup - liminf}

The liminf and limsup are commonly used expressions in mathematics. Their definitions are as follows for any sequence $\left\{x_{n}\right\}_{n \geq 0}$ :

$$
\begin{aligned}
& \liminf _{n \rightarrow \infty} x_{n}:=\sup _{n \geq 0} \inf _{m \geq n} x_{m}=\sup \left\{\inf \left\{x_{m}: m \geq n\right\}: n \geq 0\right\} \\
& \limsup _{n \rightarrow \infty} x_{n}:=\inf _{n \geq 0} \sup _{m \geq n} x_{m}=\inf \left\{\sup \left\{x_{m}: m \geq n\right\}: n \geq 0\right\}
\end{aligned}
$$

In other words, equation (1.1.1) shows how the infimum of the tail of the sequence changes and equation (1.1.2) shows how the sup of the sequence changes in the tail. It should be noted that for any sequence $\left\{x_{n}\right\}_{n \geq 0}, \lim \sup x_{n}$ and $\lim \inf x_{n}$ always exists, however the $\lim$ only exists when $\lim \sup x_{n}=\lim \inf x_{n}:=\lim x_{n}$. The Figure 1.1 explains the concept better:

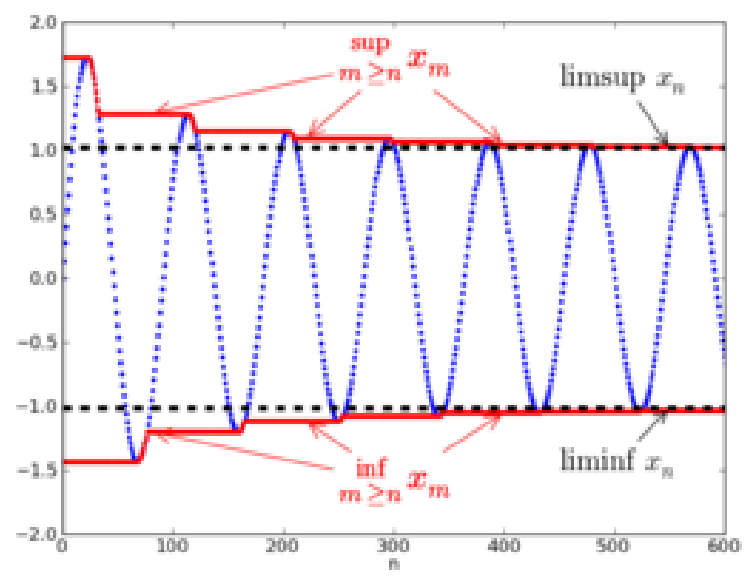

Figure 1.1: The figure showing the liminf and limsup of an oscillating function.

As explained above, the function on Figure 1.1 does not have a limit since lim sup and liminf are not equal to each other.

\subsubsection{Stochastic Processes}

A continuous time stochastic process $\left\{X_{t}\right\}_{t \geq 0}$ is a collection of random variables defined on a common probability space $(\Omega, \mathcal{F}, \mathbb{P})$. This family of random variables is indexed by the non negative real parameter $t$, which is interpreted as time. Therefore, 
$X_{t}$ can be thought as the position/value at time $t$ of a system/quantity whose evolution is random. Observe that:

- for every fixed $t \geq 0$, the function $\omega \mapsto X_{t}(\omega)$ is a random variable, while

- for every fixed $\omega \in \Omega$, the function $t \mapsto X_{t}(\omega)$ describes one possible path/trajectory of the stochastic process $\left\{X_{t}\right\}_{t \geq 0}$.

The most famous stochastic process is the Brownian Motion, which will be the focus of the following sections.

\subsubsection{Brownian Motion}

Because the thesis is about stochastic analysis, it is crucial to become familiarized with Brownian Motion $\left(B_{t}\right)$ first. It is the core of randomness in stochastic events. It would be useful to define the $B_{t}$ object mathematically as in the books [10, 16]:

Definition 1.1.1. Let $(\Omega, \mathcal{F}, \mathbb{P})$ be a probability space with a filtration $\left\{\mathcal{F}_{t}\right\}_{t \geq 0}$ (a filtration will be explained in the next section). A (standard) one-dimensional Brownian Motion is a real-valued continuous $\left\{\mathcal{F}_{t}\right\}$-adapted process $\left\{B_{t}\right\}_{t \geq 0}$ with the following properties:

1. $B_{0}=0$ a.s.;

2. for $0 \leq s<t<\infty$, the increment $B_{t}-B_{s}$ is normally distributed with mean zero and variance $t-s$;

3. for $0 \leq s<t<\infty$, the increment $B_{t}-B_{s}$ is independent of $\left\{\mathcal{F}_{s}\right\}$.

The 3 rd property can be re-worded to say that, the increments of Brownian Motion are independent of each other. Together with 2 nd definition of Brownian Motion, these properties construct the random object whose value depends on both time and chance. It is a perfect mix to develop the stochastic differential equations later as present in this thesis.

There are other important properties of Brownian Motion, which will be pointed out now. The first property is:

Theorem 1.1.2. Let $\left\{B_{t}\right\}_{t \geq 0}$ be a Brownian Motion and $\left\{\pi_{n}\right\}_{n \geq 1}$ be any sequence of finite partitions of the interval $[0, T]$ satisfying

$$
\lim _{n \rightarrow+\infty}\left|\pi_{n}\right|=0
$$

Then,

$$
\lim _{n \rightarrow+\infty} \sum_{j=1}^{N_{n}}\left(B_{t_{j}^{(n)}}-B_{t_{j-1}^{(n)}}\right)^{2}=b-a \quad \text { in } \mathbb{L}^{2}(\Omega) .
$$


Meaning:

$$
\mathbb{E}\left[\left(\sum_{j=1}^{N_{n}}\left(B_{t_{j}^{(n)}}-B_{t_{j-1}^{(n)}}\right)^{2}-(b-a)\right)^{2}\right] \stackrel{n \rightarrow \infty}{\longrightarrow} 0
$$

The next property is:

Theorem 1.1.3. Let $\left\{B_{t}\right\}_{t \geq 0}$ be a Brownian Motion. Then,

$$
\lim _{t \rightarrow \infty} \frac{B_{t}}{t}=0 \quad \text { a.s. }
$$

This equality is also known as the Law of Large Numbers for Martingales as shown in [10]. In other words, although the Brownian Motion is a stochastic process with a linearly increasing variance with respect to time, it's magnitude will almost surely be outbounded by $t$ and further reading can be done in [10.

On the other hand, the last property of Brownian Motion that will be addressed in this study is that:

Theorem 1.1.4. Let $\left\{B_{t}\right\}_{t \geq 0}$ be a Brownian Motion. Then,

$$
\mathbb{P}\left(t \mapsto B_{t} \text { is nowhere differentiable }\right)=1 .
$$

In other words, Brownian Motion $B_{t}$ is not differentiable in anywhere a.s. as shown in [10, 16]. This property of Brownian Motion made it very hard to incorporate into stochastic differential and integral equations until mid 1900s.

\subsubsection{Filtrations, Martingales and Conditional Expectation}

A filtration $\left\{\mathcal{F}_{t}\right\}_{t \geq 0}$ on the probability space $(\Omega, \mathcal{F}, \mathbb{P})$ is an increasing family of $\sigma$-algebras contained in $\mathcal{F}$, i.e.

$$
\mathcal{F}_{s} \subseteq \mathcal{F}_{t} \subseteq \mathcal{F} \quad \text { for all } 0 \leq s<t .
$$

A continuous time stochastic process $\left\{X_{t}\right\}_{t \geq 0}$ is said to be adapted to the filtration $\left\{\mathcal{F}_{t}\right\}_{t \geq 0}$ if $X_{t}$ is $\mathcal{F}_{t}$-measurable, for all $t \geq 0$. Measurability in general means that, $\left\{X_{t}^{-1}(S): S \in \sigma(\mathbb{R})\right\} \in \mathcal{F}_{t}$, because $X_{t}$ is a mapping from $[0, T] \times \Omega$ to $\mathbb{R}$.

Definition 1.1.5. The natural filtration $\left\{\mathcal{F}_{t}^{B}\right\}_{t \geq 0}$ of a Brownian Motion $\left\{B_{t}\right\}_{t \geq 0}$ is the filtration defined as

$$
\mathcal{F}_{t}^{B}:=\sigma\left(B_{s}, 0 \leq s \leq t\right), \quad t \geq 0
$$

this means that $\mathcal{F}_{t}^{B}$ is generated by events of the form $\left\{B_{s} \in A\right\}$, for $s \in[0, t]$ and $A$ being an interval of $\mathbb{R}$. 
The filtration and the size of $\sigma$-algebras are generally associated with the information one has about the system. In this case, as time passes since you know more about your random process and you obtain bigger $\mathcal{F}_{t}^{B}$ including the previous ones, hence reveal more of your $\mathcal{F}$. One can also notice that a value for $B_{s}$ is measurable for $\mathcal{F}_{t}$ for $s \leq t$, since the event $B_{s}$ already happened and it's information is already encapsulated within $\mathcal{F}_{t}$.

Definition 1.1.6. Let $X$ be a random variable in $\mathbb{L}^{2}(\Omega, \mathcal{F}, \mathbb{P})$. If $\mathcal{G}$ is a sub-sigmaalgebra of $\mathcal{F}$, we define the conditional expectation of $X$ given $\mathcal{G}$, denoted $\mathbb{E}[X \mid \mathcal{G}]$, to be the unique element of $\mathbb{L}^{2}(\Omega, \mathcal{G}, \mathbb{P})$ such that

$$
\mathbb{E}\left[|X-\mathbb{E}[X \mid \mathcal{G}]|^{2}\right] \leq \mathbb{E}\left[|X-Y|^{2}\right], \quad \text { for all } Y \in \mathbb{L}^{2}(\Omega, \mathcal{G}, \mathbb{P}) .
$$

It has been observed that $\mathbb{L}^{2}(\Omega, \mathcal{G}, \mathbb{P}) \subseteq \mathbb{L}^{2}(\Omega, \mathcal{F}, \mathbb{P})$; in fact, since $\mathcal{G} \subseteq \mathcal{F}$, the condition of being $\mathcal{G}$-measurable is more stringent than the one of being $\mathcal{F}$-measurable. Therefore, for $X \in \mathbb{L}^{2}(\Omega, \mathcal{F}, \mathbb{P})$, the conditional expectation $\mathbb{E}[X \mid \mathcal{G}]$ is the best approximation of $X$ with an element from $\mathbb{L}^{2}(\Omega, \mathcal{G}, \mathbb{P})$. Here, by best approximation we mean the element having the minimum quadratic distance from $X$.

In the sequel the space $\mathbb{L}^{2}(\Omega, \mathcal{F}, \mathbb{P})$ will be simply denoted as $\mathbb{L}^{2}(\Omega)$.

Remark 1.1.7. The list some crucial properties of the conditional expectation is below:

- if $\mathcal{G}$ is the trivial sigma-algebra $\{\varnothing, \Omega\}$, then $\mathbb{E}[X \mid \mathcal{G}]=\mathbb{E}[X]$;

- if $\alpha, \beta, \gamma \in \mathbb{R}$, then $\mathbb{E}[\alpha X+\beta Y+\gamma \mid \mathcal{G}]=\alpha \mathbb{E}[X \mid \mathcal{G}]+\beta \mathbb{E}[Y \mid \mathcal{G}]+\gamma$;

- if $X$ is $\mathcal{G}$-measurable, then $\mathbb{E}[X \mid \mathcal{G}]=X$;

- if $X$ is independent of $\mathcal{G}$, then $\mathbb{E}[X \mid \mathcal{G}]=\mathbb{E}[X]$;

- if $\mathcal{H} \subseteq \mathcal{G}$, then $\mathbb{E}[\mathbb{E}[X \mid \mathcal{G}] \mid \mathcal{H}]=\mathbb{E}[X \mid \mathcal{H}]$;

- if $X$ is $\mathcal{G}$-measurable, then $\mathbb{E}[X Y \mid \mathcal{G}]=X \mathbb{E}[Y \mid \mathcal{G}]$.

Definition 1.1.8. A continuous time stochastic process $\left\{X_{t}\right\}_{t \geq 0}$ is an $\left(\left\{\mathcal{F}_{t}\right\}_{t \geq 0}, \mathbb{P}\right)$ martingale if it is $\left\{\mathcal{F}_{t}\right\}_{t \geq 0}$-adapted, $X_{t} \in \mathbb{L}^{2}(\Omega)$, for all $t \geq 0$, and

$$
\mathbb{E}\left[X_{t} \mid \mathcal{F}_{s}\right]=X_{s}, \quad \text { for all } 0 \leq s \leq t .
$$

Any Brownian Motion $\left\{B_{t}\right\}_{t \geq 0}$ defined on the probability space $(\Omega, \mathcal{F}, \mathbb{P})$ is an $\left(\left\{\mathcal{F}_{t}^{B}\right\}_{t \geq 0}, \mathbb{P}\right)$ - martingale: in fact, for any $0 \leq s \leq t$ we have

$$
\begin{aligned}
\mathbb{E}\left[B_{t} \mid \mathcal{F}_{s}^{B}\right] & =\mathbb{E}\left[B_{t}-B_{s}+B_{s} \mid \mathcal{F}_{s}^{B}\right] \\
& =\mathbb{E}\left[B_{t}-B_{s} \mid \mathcal{F}_{s}^{B}\right]+\mathbb{E}\left[B_{s} \mid \mathcal{F}_{s}^{B}\right] \\
& =\mathbb{E}\left[B_{t}-B_{s}\right]+B_{s} \quad \text { (due to independence and measurabilty) } \\
& =B_{s} .
\end{aligned}
$$

This definition of Martingales are presented here just because the the sake of completeness, however they will not be addressed in this study frequently. 


\subsubsection{The Itô Integral}

In order to develop differential equations with stochasticity, the Brownian Motion should be added to either differential or integral equations someway but as stated earlier the main problem is that $d B_{t}$ does not exist. One solution to this problem came from Wiener, where he was able to define integrals in type $\int_{0}^{T} f(t) d B_{t}$, where $f(t)$ is a deterministic function. However in this study, the Itô integral will be used. The Itô Integral, on the other hand, is very similar to a Wiener Integral but a more general version of it. It has a distinction that, instead of $f(t)$, the integral is defined for a stochastic process $f(t, \omega)$ where $\omega$ is the chance parameter. To ease the notation, from now on $\omega$ may be omitted, and instead of $f(t, \omega), f_{t}$ may be written. However it should be understood that the chance parameter is a fundamental part of a stochastic process and it exists even though it is not reported explicitly. As already stated earlier, unlike of regular functions, a stochastic process's values change not only depending on time but also according to chance.

In order to construct such an integral, first Itô started by defining the integral with the most simplistic type of stochastic processes and built his way up to a much broader type of processes. Below his methodology are going to be explained briefly. For further reading, please refer to books [16, 10, 1].

As a start, like in [16], consider $f_{n}(t, \omega)$ to be a $\left\{\mathcal{F}_{t}\right\}_{t \geq 0}$ measurable stochastic process defined in interval $[0, T]$ and $f_{n}:=\sum_{i=1}^{n} \eta_{i-1} \mathbb{1}_{\left[t_{i-1}, t_{i}\right)}$, where $\mathbb{1}$ is the indicator function, $\eta_{i-1}$ 's are $\mathcal{F}_{t_{i-1}}$ measurable random variables belonging to $L^{2}(\Omega), t_{0}=0$ and $t_{n}=T$. In this report $L^{2}(\Omega)$ will be used to denote the Hilbert Space of square integrable random variables. Such a process is called a simple stochastic process and takes discrete random values according to $\eta_{i}$, for each partition of interval $\left[t_{i-1}, t_{i}\right)$. For such processes, define function

$$
I\left(f_{n}\right):=\sum_{i=1}^{n} \eta_{i-1}\left(B_{t_{i}}-B_{t_{i-1}}\right)
$$

There are some remarks to point out here. Firstly, it can be shown that due to the 2 nd,3 rd definitions of Brownian Motion and the $\mathcal{F}_{t_{i-1}}$ measurability the following two equations (1.1.6) and (1.1.7) hold:

$$
\mathbb{E}[I(f)]=0
$$

and 


$$
\begin{array}{rlr}
\mathbb{E}\left[\left(I\left(f_{n}\right)^{2}\right)\right] & =\mathbb{E}\left[\sum_{i, j=1}^{n} \eta_{i-1} \eta_{j-1}\left(B_{t_{i}}-B_{t_{i-1}}\right)\left(B_{t_{j}}-B_{t_{j-1}}\right)\right] \\
& =\sum_{i=1}^{n} \eta_{i-1}^{2} \mathbb{E}\left[\left(B_{t_{i}}-B_{t_{i-1}}\right)^{2}\right] & \text { (Independence and } \mathcal{F}_{t_{i-1}} \text { Measurability) } \\
& =\sum_{i=1}^{n} \eta_{i-1}^{2}\left(t_{i}-t_{i-1}\right) & \text { (Due to known variance) } \\
& =\int_{0}^{T} f_{n}^{2} d t &
\end{array}
$$

In this study $L_{a d}^{2}(\Omega \times[0, T])$ means the Hilbert Space of adapted square integrable stochastic processes in the interval $[0, T]$. Let $f$ be a process such that $f \in L^{2}(\Omega \times[0, T])$, which means $\int_{0}^{T} \mathbb{E}\left[f^{2}\right] d t<\infty$. For such processes there exists a sequence $\left\{f_{n}\right\}_{n=1}^{\infty}$, where $f_{n} \stackrel{n \rightarrow \infty}{\longrightarrow} f$ in $L^{2}(\Omega \times[0, T])$. That means:

$$
\lim _{n \rightarrow+\infty} \mathbb{E}\left[\int_{0}^{T}\left|f_{n}-f\right|^{2} d t\right]=0 .
$$

Because $f_{n}$ converges to $f$ in $L^{2}(\Omega \times[0, T])$, it can be shown that the sequence $\left\{I\left(f_{n}\right)\right\}_{n=1}^{\infty}$ is Cauchy in $L^{2}(\Omega)$. Also due to the completeness of $L^{2}(\Omega)$, it is also convergent so it can be said that:

$$
\lim _{n \rightarrow \infty} I\left(f_{n}\right)=I(f), \quad \text { in } L^{2}(\Omega)
$$

The limiting function $I(f)$ is defined to be the Itô Integral of $f$ and denoted as: $\int_{0}^{T} f d B_{t}$ and it is a continuous $\left(\left\{\mathcal{F}_{t}\right\}_{t \in[0, T]}, \mathbb{P}\right)$ martingale.

What all this means is that, even though the differential of $B_{t}$ does not exists, the Itô Integral, involving $d B_{t}$ and a stochastic process from the set $L_{a d}^{2}(\Omega \times[0, T])$, can be defined as a limiting process of a sum. It is worth to mention that the previous results (1.1.6) and (1.1.7) (hence variance) hold for Itô Integral of elements of $L_{a d}^{2}(\Omega \times[0, T])$.

Another generalization can be made to define the Itô Integral for a broader class of functions. Instead of $f \in L_{a d}^{2}(\Omega \times[0, T])$ now functions from $\mathcal{L}_{a d}\left(\Omega ; L^{2}([0, T])\right)$ will be used as the integrand. The definition of $\mathcal{L}_{a d}\left(\Omega ; L^{2}([0, T])\right)$ is given below:

Definition 1.1.9. We set

$$
\begin{array}{r}
\mathcal{L}_{a d}\left(\Omega ; L^{2}([0, T])\right):=\left\{\left\{X_{t}\right\}_{t \in[0, T]} \text { is an }\left\{\mathcal{F}_{t}\right\}_{t \in[0, T]}\right. \text {-adapted stochastic process } \\
\text { such that } \left.\mathbb{P}\left(\int_{0}^{T} X_{t}^{2} d t<+\infty\right)=1\right\}
\end{array}
$$


In words: an adapted stochastic process belongs to $\mathcal{L}_{a d}\left(\Omega ; L^{2}([0, T])\right)$ if the path $t \in$ $[0, T] \mapsto X_{t}$ is square integrable, almost surely.

Any adapted stochastic process with almost surely continuous paths belongs to $\mathcal{L}_{a d}\left(\Omega ; L^{2}([0, T])\right)$; in fact, continuous functions on the compact interval $[0, T]$ are bounded and hence square integrable:

$$
\int_{0}^{T} X_{t}^{2} d t \leq \int_{0}^{T} \sup _{t \in[0, T]}\left|X_{t}\right|^{2} d t=T \sup _{t \in[0, T]}\left|X_{t}\right|^{2}<+\infty,
$$

almost surely. In other words, it can be seen that this definition is a more general definition since if a function is continuous, it is also square integrable in a compact interval. Briefly, if the process $f(t, \omega)$ has continuous sample paths, then one can define the Itô Integral again similarly, as in [10, 16]. However in this case, the nice properties (1.1.6) and (1.1.7) are not guaranteed to be satisfied and the convergence strength of the limiting sum is weakened to in probability instead of $L^{2}(\Omega)$. If one wants to use these properties, then he/she should check if the process $f_{t}$ is in $L_{a d}^{2}(\Omega \times[0, T])$ directly.

\subsubsection{Stochastic Differential Equations (SDEs)}

Because generating stochastic integrals involving, stochastic processes as their integrands and $d B_{t}$ as the integrating measures, are already possible. In this section, the applications of this new calculus type will be investigated.

Definition 1.1.10. A stochastic differential equation of Itô-type is an identity of the form

$$
X_{t}=x+\int_{0}^{t} b\left(s, X_{s}\right) d s+\int_{0}^{t} \sigma\left(s, X_{s}\right) d B_{s}, \quad t \in[0, T]
$$

where the stochastic process $\left\{X_{t}\right\}_{t \in[0, T]}$ is the unknown, $x$ is a real number, Brownian Motion $\left\{B_{t}\right\}_{t \in[0, T]}$ are given and the functions $b\left(t, X_{t}\right)$ and $\sigma\left(t, X_{t}\right)$ satisfy that:

$$
\mathbb{P}\left(\int_{0}^{T}\left|b\left(t, X_{t}\right)\right| d t<+\infty\right)=1,
$$

and $\left\{\sigma\left(t, X_{t}\right)\right\}_{t \in[0, T]}$ belongs to $\mathcal{L}_{a d}\left(\Omega ; L^{2}([0, T])\right)$.

Equation 1.1.8) is usually rewritten as

$$
\left\{\begin{array}{l}
\left.\left.d X_{t}=b\left(t, X_{t}\right) d t+\sigma\left(t, X_{t}\right) d B_{t}, \quad t \in\right] 0, T\right] \\
X_{0}=x
\end{array}\right.
$$

symbolically. 
The differential expression is symbolic because the differential of Brownian Motion does not exists. In other words such differential expressions are short hand notations for the related integral equaitons. The functions $b$ and $\sigma$ are called drift and diffusion coefficients, respectively; $x$ is the initial condition. The integral $\int_{0}^{t} \sigma\left(s, X_{s}\right) d B_{s}$ is an Itô integral.

Definition 1.1.11. A stochastic process $\left\{X_{t}\right\}_{t \in[0, T]}$ is a strong solution of equation 1.1.8 if the following conditions are satisfied:

- $\left\{X_{t}\right\}_{t \in[0, T]}$ is $\left\{\mathcal{F}_{t}^{B}\right\}_{t \in[0, T]}$-adapted;

- $\mathbb{P}\left(\int_{0}^{T}\left|b\left(t, X_{t}\right)\right| d t<+\infty\right)=1$;

- $\mathbb{P}\left(\int_{0}^{T}\left|\sigma\left(t, X_{t}\right)\right|^{2} d t<+\infty\right)=1$;

- equation (1.1.8) is verified almost surely, for all $t \in[0, T]$.

The existence of a unique strong solution is usually guaranteed by imposing some regularity assumptions on the coefficients of the stochastic differential equation.

Definition 1.1.12. A function $f:[0, T] \times \mathbb{R} \rightarrow \mathbb{R}$ is said to be Lipschitz-continuous in the second variable if there exist a positive constant $L$ such that

$$
|f(t, x)-f(t, y)| \leq L|x-y|, \quad \text { for all } t \in[0, T] \text { and } x, y \in \mathbb{R} .
$$

A function $f:[0, T] \times \mathbb{R} \rightarrow \mathbb{R}$ is said to be locally Lipschitz-continuous in the second variable if for every $n \in \mathbb{N}$ there exist a positive constant $L_{n}$ such that

$$
|f(t, x)-f(t, y)| \leq L_{n}|x-y|, \quad \text { for all } t \in[0, T],|x| \leq n \text { and }|y| \leq n .
$$

Observe that (locally) Lipschitz-continuous are necessarily continuous,; in fact,

$$
0 \leq \lim _{y \rightarrow x}|f(t, x)-f(t, y)| \leq \lim _{y \rightarrow x} L|x-y|=0 .
$$

Remark 1.1.13. Examples of Lipschitz continuous functions are $a x+b,|x|, \sin (x)$, $\cos (x), \arctan (x), e^{-|x|}$. In general, a continuously differentiable function $f: \mathbb{R} \rightarrow \mathbb{R}$ with bounded derivative, i.e. $\left|f^{\prime}(x)\right| \leq L$, for all $x \in \mathbb{R}$ and some $L>0$, is Lipschitz continuous. In fact, by the mean value theorem, for all $x, y \in \mathbb{R}$, there exists $\eta \in[x, y]$ such that

$$
|f(x)-f(y)|=\left|f^{\prime}(\eta)\right||x-y| \leq L|x-y| .
$$

The function $\sqrt{x}$ is not Lipschitz continuous at $x=0$ but is Lipschitz continuous on the interval $] 0+\infty[$.

A continuously differentiable function $f: \mathbb{R} \rightarrow \mathbb{R}$ is locally Lipschitz continuous; in fact, by the mean value theorem, for all $n \in \mathbb{N}$ and $x, y \in \mathbb{R}$ with $|x|,|y| \leq n$, there exists $\eta_{n} \in[x, y]$ such that

$$
|f(x)-f(y)|=\left|f^{\prime}\left(\eta_{n}\right)\right||x-y| \leq \max _{|z| \leq n}\left|f^{\prime}(z)\right||x-y|=L_{n}|x-y| .
$$


Definition 1.1.14. A function $f:[0, T] \times \mathbb{R} \rightarrow \mathbb{R}$ is said to satisfy the linear growth condition in the second variable if there exist a positive constant $M$ such that

$$
|f(t, x)| \leq M(1+|x|), \quad \text { for all } t \in[0, T] \text { and } x \in \mathbb{R} .
$$

Example 1.1.15. The Lipschitz continuous functions $a x+b,|x|, \sin (x), \cos (x), \arctan (x)$, $e^{-|x|}$ from Example 1.1 .13 satisfy also the linear growth condition. The functions $x^{2}$ and $e^{x}$ are locally Lipschitz continuous but do not satisfy the linear growth condition. The bounded function $f(x)=2 \cdot \mathbb{1}_{[0,1]}(x)+3 \cdot \mathbb{1}_{[2,4]}(x)$ satisfies the linear growth condition but it is not Lipschitz continuous (since it is discontinuous).

Theorem 1.1.16 (Existence and uniqueness). Assume that the functions $b, \sigma:[0, T] \times$ $\mathbb{R} \rightarrow \mathbb{R}$ are Lipschitz continuous and satisfy the linear growth condition in the second variable. Then, the stochastic differential equation

$$
\left\{\begin{array}{l}
\left.\left.d X_{t}=b\left(t, X_{t}\right) d t+\sigma\left(t, X_{t}\right) d B_{t}, \quad t \in\right] 0, T\right] \\
X_{0}=x
\end{array}\right.
$$

admits a unique continuous strong solution $\left\{X_{t}\right\}_{t \in[0, T]}$.

However it is woth to note that, having coefficients Lipschitz continuous and satisfying the linear growth condition is only sufficient for having a unique strong solution, and not necessary. That means there are SDEs not satisfying these conditions, nevertheless they admit a unique strong solution.

Moreover, the most crucial thing to know about SDE's is the Itô's Formula. It is the basis of this calculus type and it will be used very frequently to solve SDEs.

Itô's Formula: For a continuous function $\theta(t, x)$ with continuous partial derivatives $\frac{\partial \theta}{\partial t}, \frac{\partial \theta}{\partial x}$ and $\frac{\partial^{2} \theta}{\partial x^{2}}$ and an Ito process $X_{t}$ as in the definition 1.1 .10 , with the general equation $d X_{t}=f\left(t, X_{t}\right) d B_{t}+g\left(t, X_{t}\right) d t$, the following expression holds:

$$
d \theta\left(t, X_{t}\right)=\left.\frac{\partial \theta(t, x)}{\partial t}\right|_{x=X_{t}} d t+\left.\frac{\partial \theta(t, x)}{\partial x}\right|_{x=X_{t}} d X_{t}+\left.\frac{1}{2} \frac{\partial^{2} \theta(t, x)}{\partial x^{2}}\right|_{x=X_{t}} d X_{t}^{2}
$$

in differential form. Or

$$
\begin{aligned}
\theta\left(T, X_{T}\right)=\theta\left(0, X_{0}\right) & +\int_{0}^{T} \frac{\partial \theta}{\partial x}\left(t, X_{t}\right) f\left(t, X_{t}\right) d B_{t} \\
& +\int_{0}^{T}\left[\frac{\partial \theta}{\partial t}\left(t, X_{t}\right)+\frac{\partial \theta}{\partial x}\left(t, X_{t}\right) g\left(t, X_{t}\right)+\frac{1}{2} \frac{\partial^{2} \theta}{\partial x^{2}}\left(t, X_{t}\right) f\left(t, X_{t}\right)\right] d t
\end{aligned}
$$

in integral form.

When the expression 1.1 .9 is used in a smart way, it can be very useful to solve SDEs. One particular example will be used in the next section called "The OrnsteinUhlenbeck (O-U) Process". 


\section{The Ornstein-Uhlenbeck (O-U) Process}

The O-U process is a well studied type of SDE in literature. It is the solution of the following Langevin equaiton:

$$
d X_{t}=\alpha X_{t} d t+\sigma d B_{t}, \quad X_{0}=0
$$

where $\alpha$ and $\sigma$ are real coefficients, hence they are Lipschitz Continuous and they satisfy the linear growth condition, so that by Theorem 1.1.16 leads to having a unique strong solution to the equation (1.1.11) by [10, and the choice of initial value was arbitrary. Now through Itô's Formula it will be shown that the solution is:

$$
\sigma e^{\alpha t} \int_{0}^{t} e^{-\alpha s} d B_{s} .
$$

Firstly, let $Y_{t}:=\int_{0}^{t} e^{-\alpha s} d B_{s}$, so that

$$
d Y_{t}=e^{-\alpha t} d B_{t} \quad \text { and } \quad X_{t}=\sigma e^{\alpha t} Y_{t} .
$$

In order to use Itô's Formula (1.1.9), one should define a function $\theta(t, x)$ that is continuous and has continuous partial derivatives $\frac{\partial \theta}{\partial t}$, $\frac{\partial \theta}{\partial x}$ and $\frac{\partial^{2} \theta}{\partial x^{2}}$. Let $\theta(t, x):=\sigma e^{\alpha t} x$, so that $\frac{\partial \theta}{\partial t}=\sigma \alpha e^{\alpha t} x, \frac{\partial \theta}{\partial x}=\sigma e^{\alpha t}$ and $\frac{\partial^{2} \theta}{\partial x^{2}}=0$. Hence according to expression (1.1.9), the differential becomes:

$$
d \theta\left(t, Y_{t}\right)=\sigma \alpha e^{\alpha t} Y_{t} d t+\sigma e^{\alpha t} d Y_{t}
$$

One can notice using identities 1.1 .13 on 1.1 .14 it is found that:

$$
d \theta\left(t, Y_{t}\right)=d X_{t}=\alpha X_{t} d t+\sigma d B_{t} .
$$

Which is the Langevin equation provided in (1.1.11). Because the Itô Formula is used on the expression (1.1.12 and it produced the SDE of interest, it must be the unique strong solution. The important remarks about this solution is, the expectation and variance of expression (1.1.12). They can be calculated as explained in (1.1.6) and (1.1.7).

$$
\begin{aligned}
& \mathbb{E}\left[\sigma e^{\alpha t} \int_{0}^{t} e^{-\alpha s} d B_{s}\right]=0 \text { and } \\
& \mathbb{V}\left[\sigma e^{\alpha t} \int_{0}^{t} e^{-\alpha s} d B_{s}\right]=\sigma^{2} e^{2 \alpha t} \int_{0}^{t} e^{-2 \alpha s} d s=\frac{\sigma^{2}}{2 \alpha}\left(1-e^{-2 \alpha t}\right)
\end{aligned}
$$

These properties of make $\mathrm{O}-\mathrm{U}$ processes a very handy option for deterministic models to perturb into. Because the expectation is 0, it means on average you don't deviate from the deterministic model. Moreover if you want to add a finite amount of variance, it is a better choice than $B_{t}$ since $\lim _{t \rightarrow \infty} \mathbb{V}\left[B_{t}\right]=\lim _{t \rightarrow \infty} t=\infty$ but $\lim _{t \rightarrow \infty} \mathbb{V}\left[\sigma^{2} /(2 \alpha)\left(1-e^{-2 \alpha t}\right)\right]=\sigma^{2} /(2 \alpha)$, hence one can play with parameters $\sigma$ and $\alpha$ to adjust the amount of variability he/she wants to add to the deterministic model. 


\subsubsection{Wong-Zakai Theorem}

The parameter perturbation method, it will be explained later in this study deeper, is a common method to make a deterministic ordinary differential equation (ODE) to a stochastic one by adding a stochastic process to the ODE. The Wong-Zakai Theorem, is one of the techniques to carry-on this transition, without directly modifying the equation with delicate objects like the differential of Brownian Motion or the differential of other stochastic processes.

Instead, as shown [13], the "smoother" approximations of such processes, which are differentiable can be used to modify the ODE. One example that will be used in this study is the polygonal approximation of Brownian Motion $\left\{B_{t}^{n}\right\}_{t \geq 0}$, where $n$ denotes the number of sample points in the partition of the interval $[0, T]$. This is a random function where " $n$ " sample points of Brownian Motion are joined linearly, forming a piecewise continuous and piecewise differentiable approximation of Brownian Motion. It should be also noted that $B_{t}^{n}$ converges to $B_{t}$ uniformly as $n \rightarrow \infty$, as also stated in [2].

This approximation can be used to modify the original deterministic ODE without any problem because, although the Brownian Motion is nowhere differentiable, $B_{t}^{n}$ is. Later when the limit of the approximated differential equation is taken, by Wong-Zakai Theorem, the result converges to the Stratonovich Stochastic Integral [13].

\section{Converting Stratonovich SDEs to Itô}

Because converting Stratonovich Integrals to Itô is no problem, one can also obtain SDE's in Itô sense by using Wong-Zakai approach on ODEs. In other words:

$$
\frac{d f^{n}(t)}{d t}=g(t, f(t))+\sigma(f(t)) \frac{d B_{t}^{n}}{d t} \frac{n \rightarrow \infty}{W-Z T h} d f(t)=g(t, f(t)) d t+\sigma(f(t)) \circ d B_{t}
$$

where the symbol "o" represents the type of SDE as Stratonovich. In order to convert (1.1.16), a Stratonovich SDE, to an Itô one, the following substitution should be used:

$$
\sigma(f(t)) \circ d B_{t}=\sigma(f(t)) \frac{1}{2} \frac{d \sigma}{d f}(f(t)) d t+\sigma(f(t)) d B_{t}
$$

which makes equation 1.1 .16 to be:

$$
d f(t)=g(t, f(t)) d t+\sigma(f(t)) \circ d B_{t}=\left[g(t, f(t))+\sigma(f(t)) \frac{1}{2} \frac{d \sigma}{d f}(f(t))\right] d t+\sigma(f(t)) d B_{t} .
$$

One immediate remark is that, if $\sigma$ is independent of $f(t)$, then Stratonovich and Itô type of SDEs result in the same expression. This type of conversion will be used in the thesis in the following chapters.

\subsection{The SIS Model}

There are key biological parameters to analyse in epidemiology. The pathogen's type, infection target, pathogen's vulnerability to immune system, contagiousness and 
its life time outside of the host are certainly some of these key parameters. However, they are not enough to fully describe how an epidemic evolves. A key element, which makes epidemiology a very complex area, is the interaction inside the population and its unpredictability.

To address this feature several mathematical models have been proposed and analysed in the literature: see e.g. [6, 5, 15, 7] and the references quoted there. In the classic susceptible-infected-susceptible (SIS) model there is no long term immunity for the infection. One example of such case is Gonorrhea, as described in [6], where individuals who are recovered from the infection become infected again and again. This means that long term immunity is not effective in prevention from re-infection.

From a mathematical point of view, the SIS model (see e.g. [3]) is a very handy tool that describes the average evolution of an infection with no immunity. It consists of the system of ordinary differential equations

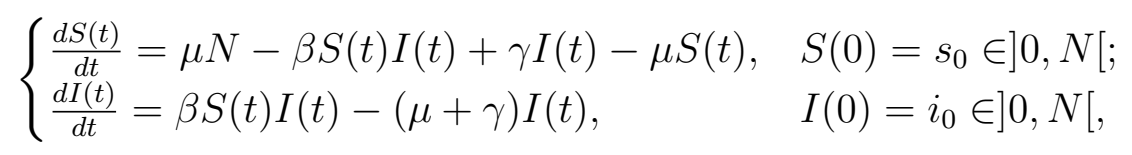

where $S(t)$ and $I(t)$ denote the number of susceptibles and infecteds at time $t$, respectively. Here, $N:=s_{0}+i_{0}$ is the initial size of the population amongst whom the disease is spreading, $\mu$ denotes the per capita death rate, $\gamma$ is the rate at which infected individuals become cured and $\beta$ stands for the disease transmission coefficient. If we sum the equations in (1.2.1), we get that

$$
\frac{d}{d t}(S(t)+I(t))=\mu(N-(S(t)+I(t))), \quad S(0)+I(0)=N,
$$

which yields

$$
S(t)+I(t)=S(0)+I(0)=N, \quad \text { for all } t \geq 0 .
$$

Therefore, system (1.2.1) can be reduced to the differential equation

$$
\left.\frac{d I(t)}{d t}=\beta I(t)(N-I(t))-(\mu+\gamma) I(t), \quad I(0)=i_{0} \in\right] 0, N[,
$$

with $S(t):=N-I(t)$. Furthermore, equation 1.2 .2$)$ can be solved explicitly as

$$
I(t)=\frac{i_{0} e^{[\beta N-(\mu+\gamma)] t}}{1+\beta \int_{0}^{t} i_{0} e^{[\beta N-(\mu+\gamma)] s} d s}, \quad t \geq 0 .
$$

This explicit representation easily identifies two different asymptotic regimes for the solution $I(t)$, namely

$$
\lim _{t \rightarrow+\infty} I(t)= \begin{cases}0, & \text { if } R_{0}^{D} \leq 1 \\ N\left(1-\frac{1}{R_{0}^{D}}\right), & \text { if } R_{0}^{D}>1\end{cases}
$$


where

$$
R_{0}^{D}:=\frac{\beta N}{\mu+\gamma}
$$

This ratio is known as basic reproduction number of the infection and determines whether the disease will become extinct, i.e. $I(t)$ will tend to zero as $t$ goes to infinity, or will be persistent, i.e. $I(t)$ will tend to a positive limit as $t$ increases.

\subsection{Perturbing The SIS Model In Literature}

As seen from (1.2.3) and identity $S(t)=N-I(t)$, the infected and susceptible populations will have smooth flows to each other with rates specified by the model. This description is very good at yielding overall results for a population but it fails to capture its heterogeneity, hence realism. In reality, individuals have different recovering rates or get infected at different rates, thus creating a distortion in the smooth flow between the two populations. To describe this randomness, several approaches have been proposed in the literature.

One example is the approach of Allen [1]: here one starts with a discrete Markov chain whose transition probabilities reflect the dynamical behaviour of the deterministic model; then, via a suitable scaling on the one-step transition probability, one obtains a forward Fokker-Planck equation which is canonically associated with a stochastic differential equation.

Another common method for introducing stochasticity is the so-called parameter perturbation approach [5, 14, 4]: it amounts at perturbing one of the parameters of the model equation with a suitable source of randomness, usually a Gaussian white noise. One of the most representative papers in this direction is [5]: here the authors formally perturb equation 1.2 .2 , rewritten in the form

$$
\left.d I(t)=\beta I(t)(N-I(t)) d t-(\mu+\gamma) I(t) d t, \quad I(0)=i_{0} \in\right] 0, N[,
$$

through the replacement

$$
\beta d t \mapsto \beta d t+\sigma d B_{t}
$$

with $\left\{B_{t}\right\}_{t \geq 0}$ being a standard one dimensional Brownian Motion and $\sigma$ an additional parameter of the model. Since $\beta$ is a parameter for disease transmission rate, the term $\beta d t$ can be interpreted as number of transmissions in time interval $[t, t+d t]$, as stated in [5]. This way, the authors propose the model

$$
d I(t)=[\beta I(t)(N-I(t))-(\mu+\gamma) I(t)] d t+\sigma I(t)(N-I(t)) d B(t),
$$

interpreted as an Itô-type stochastic differential equation, which will encapsulate the randomness in the disease transmission; moreover, they identify a stochastic reproduction number

$$
R_{0}^{S}:=R_{0}^{D}-\frac{\sigma^{2} N^{2}}{2(\mu+\gamma)}
$$


which, in contrast to 1.2 .4 , characterizes the following asymptotic behaviours:

- if $R_{0}^{S}<1$ and $\sigma^{2}<\frac{\beta}{N}$ or if $\sigma^{2}>\max \left\{\frac{\beta}{N}, \frac{\beta^{2}}{2(\mu+\gamma)}\right\}$, then the disease will become extinct, i.e.

$$
\lim _{t \rightarrow+\infty} I(t)=0
$$

- if $R_{0}^{S}>1$, then the disease will be persistent, i.e.

$$
\liminf _{t \rightarrow+\infty} I(t) \leq \xi \leq \limsup _{t \rightarrow+\infty} I(t)
$$

where $\xi:=\frac{1}{\sigma^{2}}\left(\sqrt{\beta^{2}-2 \sigma^{2}(\mu+\gamma)}-\left(\beta-\sigma^{2} N\right)\right)$.

(see also [14]). It is worth mentioning that going from 1.2 .2 to 1.3 .2 , as described in [5], one has to accept some reasonable but heuristic manipulations of the infinitesimal quantities $d t$ and $d B_{t}$. 


\section{Chapter 2}

\section{The Analysis Performed In The Thesis}

The aim of this paper is to propose a different method for perturbing the disease transmission rate in the SIS model $(1.2 .2)$. Our idea stems from the following simple observation: if we let $\beta$ in 1.2 .2 to be a function of time, then the solution formula 1.2.3 takes the form

$$
\begin{aligned}
I(t) & =\frac{i_{0} e^{N \int_{0}^{t} \beta(s) d s-(\mu+\gamma) t}}{1+i_{0} \int_{0}^{t} \beta(s) e^{N \int_{0}^{s} \beta(r) d r-(\mu+\gamma) s} d s} \\
= & \frac{i_{0} e^{N \int_{0}^{t} \beta(s) d s-(\mu+\gamma) t}}{1+\frac{i_{0}}{N}\left(e^{N \int_{0}^{t} \beta(r) d r-(\mu+\gamma) t}-1+\int_{0}^{t} e^{N \int_{0}^{s} \beta(r) d r-(\gamma+\mu) s}(\gamma+\mu) d s\right)}
\end{aligned}
$$

where in the second equality we performed an integration by parts in the denominator. Equation 2.0.1 now depends on the function $\beta(t)$ only through its integral $\int_{0}^{t} \beta(s) d s$. In [2] the authors utilized this approach to mimic the perturbation proposed in [5]; in this case, the singular perturbation

$$
\beta(t) \mapsto \beta(t)+\sigma \frac{d B_{t}}{d t},
$$

formally employed on the differential equation 1.2 .2 by the authors in [5], becomes the well defined transformation

$$
\int_{0}^{t} \beta(s) d s \mapsto \int_{0}^{t} \beta(s) d s+\sigma B_{t},
$$

if directly applied on the explicit solution (2.0.1). As shown in [2], this different procedure of parameter perturbation results in an alternative stochastic SIS model which surprisingly exhibits the same asymptotic regimes of its deterministic counterpart (1.2.2). It is important to remark that this new parameter perturbation approach, which directly 
acts on the explicit solution 2.0.1), is also justified via Wong-Zakai theorem at the level of differential equations, thus ruling out the necessity of having a closed form expression for the solution.

In the current paper we employ the just mentioned approach to the case where the perturbation is modelled as a mean reverting Ornstein -Uhlenbeck process. This choice is suggested, but not investigated, both in [1] and [5]. From a modelling point of view it is motivated by the fact that the variance of a mean reverting Ornstein Uhlenbeck process is bounded in time, while the one of a Brownian Motion, utilized in [5], is not. This feature seems to be more realistic and hence desirable. However, from a mathematical point of view, the perturbation with a mean reverting Ornstein -Uhlenbeck process makes the analysis of the model more demanding since in this case equation 1.3 .2 becomes a stochastic differential equation with random coefficients.

This problem is discussed in [4] with an approach that follows [5]. Here, we introduce the model working directly on the explicit representation (2.0.1) and cross-validate the proposal from a differential equations' perspective passing through the Wong-Zakai theorem. We prove that our model fulfils some basic biological constraints, i.e. the solution is global and lives in the interval $] 0, N[$ with probability one. Then, we analyse the asymptotic behaviour and discover that the threshold for the different regimes coincides with the one for the deterministic SIS model; in other words, the parameters describing the mean reverting Ornstein -Uhlenbeck process do not play any role in the limiting behaviour of the solution. We also identify a class of perturbations for which this invariance is preserved thus offering a complete analysis of our approach.

The paper is organized as follows: in Section 2.1 the model and its cross-validation via the Wong-Zakai theorem are introduced; Section 2.2 is devoted to the analysis of our model: support of the solution, extinction, persistence and discussion of several numerical simulations. Lastly, in Chapter 3 we address the problem of finding a general class of perturbations for which the results from Section 2.2 remain the same; numerical simulations are also presented for this enlarged framework.

\subsection{Stochastic parameter perturbation with a mean reverting Ornstein-Uhlenbeck process}

Let $\left\{Y_{t}\right\}_{t \geq 0}$ be a mean reverting Ornstein-Uhlenbeck process driven by a standard one dimensional Brownian Motion $\left\{B_{t}\right\}_{t \geq 0}$; this means that $\left\{Y_{t}\right\}_{t \geq 0}$ is the unique strong solution of the stochastic differential equation

$$
d Y_{t}=-\alpha Y_{t} d t+\sigma d B_{t}, \quad Y_{0}=0
$$

where the parameters $\alpha$ and $\sigma$ are positive real number. the process $\left\{Y_{t}\right\}_{t \geq 0}$ can be explicitly represented as

$$
Y_{t}=\sigma \int_{0}^{t} e^{-\alpha(t-s)} d B_{s}, \quad t \geq 0
$$


entailing that $Y_{t}$ is a Gaussian random variable with mean zero and variance $\frac{1-e^{-2 \alpha t}}{2 \alpha} \sigma^{2}$. The ergodic property of $\left\{Y_{t}\right\}_{t \geq 0}$ is also recalled:

$$
\lim _{t \rightarrow+\infty} \frac{1}{t} \int_{0}^{t} Y_{s} d s=0 \quad \text { almost surely. }
$$

We now perturb (2.0.1) via the substitution

$$
\beta(t) \mapsto \beta+\frac{d Y_{t}}{d t}
$$

or more rigorously

$$
\int_{0}^{t} \beta(s) d s \mapsto \int_{0}^{t}\left(\beta+\frac{d Y_{s}}{d s}\right) d s=\beta t+Y_{t}
$$

This gives

$$
\mathrm{I}_{t}:=\frac{i_{0} e^{\nu t+N Y_{t}}}{1+\frac{i_{0}}{N}\left(e^{\nu t+N Y_{t}}-1+\int_{0}^{t} e^{\nu s+N Y_{s}}(\gamma+\mu) d s\right)}, \quad t \geq 0,
$$

where to ease the notation we set $\nu:=N \beta-(\gamma+\mu)$ and $\mathrm{I}_{t}$ instead of $I\left(t, Y_{t}\right)$; note that $R_{0}^{D} \leq 1$ is equivalent to $\nu \leq 0$. The stochastic process (2.1.6) is the object of our investigation. Observe that an application of the Itô formula gives

$$
d \mathrm{I}_{t}=\left[\mathrm{I}_{t}\left(N-\mathrm{I}_{t}\right)\left(\frac{\nu}{N}-\alpha Y_{t}+\frac{N-2 \mathrm{I}_{t}}{2} \sigma^{2}\right)-\frac{\gamma+\mu}{N} \mathrm{I}_{t}^{2}\right] d t+\sigma \mathrm{I}_{t}\left(N-\mathrm{I}_{t}\right) d B_{t}
$$

This equation can be considered either as a one dimensional stochastic differential equation with random coefficients (for the presence of $\left\{Y_{t}\right\}_{t \geq 0}$ ) or, if coupled with (2.1.1), as a two dimensional system of stochastic differential equations. The local Lipschitz continuity of the coefficients of such system entails path-wise uniqueness and hence that the couple $\left\{\left(\mathrm{I}_{t}, Y_{t}\right)\right\}_{t \geq 0}$, with $\mathrm{I}_{t}$ defined in (2.1.6) and $Y_{t}$ defined in (2.1.2), is its unique solution (see e.g. Theorem 2.5, Chapter 5 in [8]).

\subsubsection{Cross-validation of the model via Wong-Zakai theorem}

We obtained the stochastic process (2.1.6) perturbing the explicit solution 2.0.1) with the transformation (2.1.5). One can however derive the stochastic differential equation (2.1.7), which is uniquely solved by (2.1.6), through a parameter perturbation procedure acting on the deterministic equation (1.2.2), which resembles the approach employed in [5].

Let $\left\{B_{t}^{\pi}\right\}_{t \in[0, T]}$ be the polygonal approximation of the Brownian Motion $\left\{B_{t}\right\}_{t \in[0, T]}$, relative to the partition $\pi$. This means that $\left\{B_{t}^{\pi}\right\}_{t \in[0, T]}$ is a continuous piecewise linear 
random function converging to $\left\{B_{t}\right\}_{t \in[0, T]}$ almost surely and uniformly on $[0, T]$, as the mesh of the partition tends to zero. Now, replace (2.1.1) with

$$
\frac{d Y_{t}^{\pi}}{d t}=-\alpha Y_{t}^{\pi}+\sigma \frac{d B_{t}^{\pi}}{d t}, \quad Y_{0}^{\pi}=0
$$

which gives a smooth approximation of $\left\{Y_{t}\right\}_{t \geq 0}$. Using $\left\{Y_{t}^{\pi}\right\}_{t \geq 0}$ instead of $\left\{Y_{t}\right\}_{t \geq 0}$ allows for a rigorous implementation of the transformation (2.1.4) in 1.2 .2 , that means

$$
\begin{aligned}
\frac{d \mathrm{I}_{t}^{\pi}}{d t}= & \beta \mathrm{I}_{t}^{\pi}\left(N-\mathrm{I}_{t}^{\pi}\right)-(\mu+\gamma) \mathrm{I}_{t}^{\pi}+\mathrm{I}_{t}^{\pi}\left(N-\mathrm{I}_{t}^{\pi}\right) \frac{d Y_{t}^{\pi}}{d t} \\
= & \beta \mathrm{I}_{t}^{\pi}\left(N-\mathrm{I}_{t}^{\pi}\right)-(\mu+\gamma) \mathrm{I}_{t}^{\pi}-\alpha \mathrm{I}_{t}^{\pi}\left(N-\mathrm{I}_{t}^{\pi}\right) Y_{t}^{\pi} \\
& +\sigma \mathrm{I}_{t}^{\pi}\left(N-\mathrm{I}_{t}^{\pi}\right) \frac{d B_{t}^{\pi}}{d t} .
\end{aligned}
$$

According to the Wong-Zakai Theorem [13], [11] the unique solution $\left\{\mathrm{I}_{t}^{\pi}\right\}_{t \in[0, T]}$ of the random ordinary differential equation (2.1.9) converges, as the mesh of the partition $\pi$ tends to zero, to the solution of the Stratonovich stochastic differential equation

$$
d \mathrm{I}_{t}=\left[\beta \mathrm{I}_{t}\left(N-\mathrm{I}_{t}\right)-(\mu+\gamma) \mathrm{I}_{t}-\alpha \mathrm{I}_{t}\left(N-\mathrm{I}_{t}\right) Y_{t}\right] d t+\sigma \mathrm{I}_{t}\left(N-\mathrm{I}_{t}\right) \circ d B_{t},
$$

which in turn is equivalent to the Itô SDE

$$
\begin{aligned}
d \mathrm{I}_{t}= & {\left[\beta \mathrm{I}_{t}\left(N-\mathrm{I}_{t}\right)-(\mu+\gamma) \mathrm{I}_{t}-\alpha \mathrm{I}_{t}\left(N-\mathrm{I}_{t}\right) Y_{t}+\frac{\sigma^{2}}{2} \mathrm{I}(t)(N-\mathrm{I}(t))(N-2 \mathrm{I}(t))\right] d t } \\
& +\sigma \mathrm{I}_{t}\left(N-\mathrm{I}_{t}\right) d B_{t} .
\end{aligned}
$$

The stochastic differential equation above coincides with (2.1.7) thus validating our parameter perturbation approach also from a model equation pont of view.

\section{$2.2 \quad$ Analysis of the stochastically perturbed SIS Model}

In this section we analyse the stochastic process $(2.1 .6)$ which we recall to be the unique strong solution of the SDE (2.1.7). we will in particular show that such process lives in the interval ]0, $N$ [, for all $t \geq 0$, almost surely and we will provide sufficient conditions for extinction and persistence.

\subsubsection{Support of the solution}

We start with the following.

Proposition 2.2.1. For the stochastic process $\left\{\mathrm{I}_{t}\right\}_{t \geq 0}$ defined in (2.1.6) we have

$$
\mathbb{P}\left(\mathrm{I}_{t} \in\right] 0, N[)=1, \quad \text { for all } t \geq 0 \text {. }
$$


Proof. First of all, we observe that $\mathrm{I}_{t}$ can be rewritten as

$$
\mathrm{I}_{t}=\frac{N i_{0} e^{\nu t+N Y_{t}}}{N-i_{0}+i_{0} e^{\nu t+N Y_{t}}+i_{0} \int_{0}^{t} e^{\nu s+N Y_{s}}(\gamma+\mu) d s} .
$$

Since by assumption $0<i_{0}<N$, it can be seen that $\mathrm{I}_{t}$ is a ratio of almost sure positive quantities; this yields $\mathrm{I}_{t}>0$ for all $t \geq 0$ almost surely. On the other hand, the last identity also gives

$$
\begin{aligned}
\mathrm{I}_{t} & <\frac{N i_{0} e^{\nu t+N Y_{t}}}{i_{0} e^{\nu t+N Y_{t}}+i_{0} \int_{0}^{t} e^{\nu s+N Y_{s}}(\gamma+\mu) d s} \\
& =\frac{N}{1+(\gamma+\mu) \int_{0}^{t} e^{\nu(s-t)+N\left(Y_{s}-Y_{t}\right)} d s} \\
& <N .
\end{aligned}
$$

The proof is complete.

\subsubsection{Extinction of the infection}

We now provide a sufficient condition for extinction; remarkably, the parameters describing the stochastic perturbation, i.e. $\alpha$ and $\sigma$, do not play role in that.

Theorem 2.2.2. If $R_{0}^{D}=\frac{\beta N}{\gamma+\mu} \leq 1$, or equivalently $\nu=\beta N-(\gamma+\nu) \leq 0$, then

$$
\lim _{t \rightarrow \infty} \mathrm{I}_{t}=0 \quad \text { almost surely. }
$$

Proof. We take $G(x):=\ln \left(\frac{x}{N-x}\right)$ for $\left.x \in\right] 0, N[$ and observe that $G$ is a strictly increasing function that maps the interval $] 0, N[$ into $]-\infty,+\infty[$. An application of the Itô formula gives

$$
d G\left(\mathrm{I}_{t}\right)=\left[\nu-N \alpha Y_{t}-(\gamma+\mu) \frac{\mathrm{I}_{t}}{N-\mathrm{I}_{t}}\right] d t+\sigma N d B_{t}
$$

which corresponds to the integral equation

$$
G\left(\mathrm{I}_{t}\right)=\ln \left(\frac{i_{0}}{N-i_{0}}\right)-\alpha N \int_{0}^{t} Y_{s} d s+\int_{0}^{t} f\left(\mathrm{I}_{s}\right) d s+\sigma N B_{t}
$$

where

$$
\left.f(x):=\nu-(\gamma+\mu) \frac{x}{N-x}, \quad \text { for } x \in\right] 0, N[.
$$


It is useful to note that $f$ is monotone decreasing on the interval $] 0, N[$ and that $f(x)<\nu$, for $x \in] 0, N[$. Therefore, from equation 2.2.1 we get

$$
\begin{aligned}
\limsup _{t \rightarrow \infty} \frac{1}{t} G\left(\mathrm{I}_{t}\right) \leq & \limsup _{t \rightarrow \infty} \frac{1}{t} \ln \left(\frac{i_{0}}{N-i_{0}}\right)-\lim _{t \rightarrow \infty} \frac{\alpha N}{t} \int_{0}^{t} Y_{s} d s \\
& +\limsup _{t \rightarrow \infty} \frac{1}{t} \int_{0}^{t} f\left(\mathrm{I}_{s}\right) d s+\sigma N \limsup _{t \rightarrow \infty} \frac{B_{t}}{t} .
\end{aligned}
$$

By recalling (2.1.3), it is easy to notice that the first two terms in right hand side above are equal to zero. Moreover, by the strong law of large numbers for martingales, Theorem 1.1.3 in this document, (see for instance [10]) we also have

$$
\lim _{t \rightarrow \infty} \frac{B_{t}}{t}=0, \quad \text { almost surely. }
$$

Therefore, inequality 2.2.3 now reads

$$
\limsup _{t \rightarrow \infty} \frac{1}{t} G\left(\mathrm{I}_{t}\right) \leq \limsup _{t \rightarrow \infty} \frac{1}{t} \int_{0}^{t} f\left(\mathrm{I}_{s}\right) d s<\nu,
$$

that means

$$
\limsup _{t \rightarrow \infty} \frac{1}{t} \ln \left(\frac{\mathrm{I}_{t}}{N-\mathrm{I}_{t}}\right)<0, \quad \text { almost surely. }
$$

Since the last statement implies our thesis, the proof is complete.

In the stochastic SIS model obtained by this parameter perturbation method from the deterministic one, the limiting behaviour of both models are the same for $R_{0}^{D} \leq 1$.

\subsubsection{Persistence of the Infection}

We now turn to the problem of finding sufficient condition for persistence of the disease. Again, the parameters describing the stochastic perturbation, i.e. $\alpha$ and $\sigma$, do not influence the threshold.

Theorem 2.2.3. If $R_{0}^{D}>1$, or equivalently $\nu>0$, then we have with probability one

$$
\limsup _{t \rightarrow \infty} \mathrm{I}_{t} \geq x^{*} \quad \text { and } \quad \liminf _{t \rightarrow \infty} \mathrm{I}_{t} \leq x^{*},
$$

where $x^{*}=N\left(1-\frac{1}{R_{0}^{D}}\right)$. 


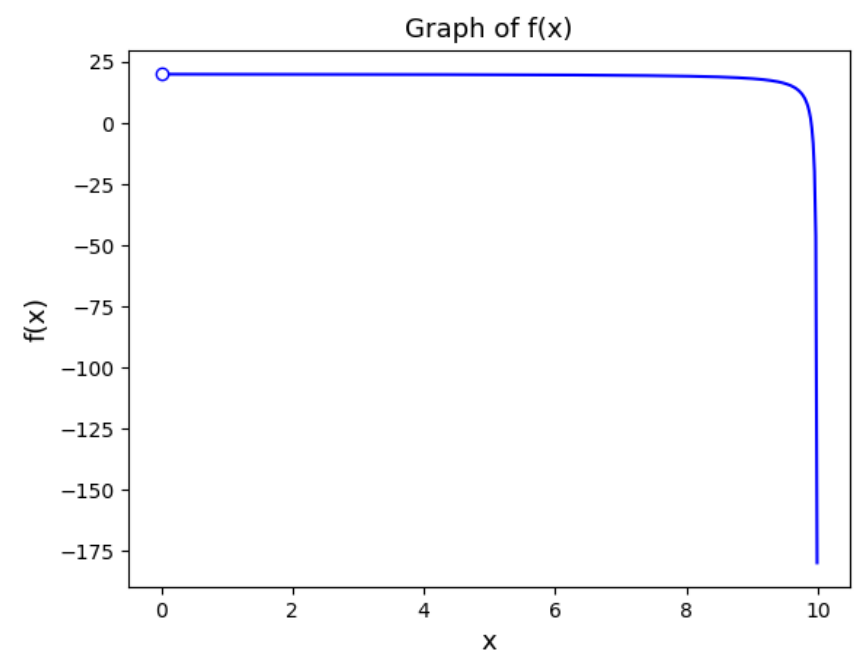

Figure 2.1: The plot of $f(x)$ from $(2.2 .2$ with $\nu=20, \gamma+\mu=0.2$ and $N=10$

Proof. The assumption of the theorem implies that the function in 2.2 .2 has a unique root in $] 0, N\left[\right.$ given by $x^{*}=N\left(1-1 / R_{0}^{D}\right)$, as shown in figure 2.1 .

We follow the proof of Theorem 5.1 in [5]. Assume the first inequality in (2.2.4) to be false. Then, there exists $\varepsilon>0$ such that

$$
\mathbb{P}\left(\Omega_{1}\right)>\varepsilon \quad \text { where } \quad \Omega_{1}:=\left\{\limsup _{t \rightarrow \infty} \mathrm{I}_{t} \leq x^{*}-\varepsilon\right\} .
$$

Therefore, for all $\omega \in \Omega_{1}$ there exists $T(\omega) \geq 0$ such that

$$
\mathrm{I}_{t} \leq x^{*}-\varepsilon, \quad \text { for all } t \geq T(\omega),
$$

and the monotonicity of $f$ yields

$$
f\left(\mathrm{I}_{t}\right) \geq f\left(x^{*}-\varepsilon\right)>0, \quad \text { for all } t \geq T(\omega) .
$$

Therefore, using identities 2.2.1), 2.1.3 and the strong law of large numbers for martingales (Theorem 1.1.3), we can write for all $\omega \in \Omega_{1}$ that

$$
\begin{aligned}
\liminf _{t \rightarrow \infty} \frac{1}{t} G\left(\mathrm{I}_{t}\right) \geq & \liminf _{t \rightarrow \infty} \frac{1}{t} \ln \left(\frac{i_{0}}{N-i_{0}}\right)-\lim _{t \rightarrow \infty} \frac{\alpha N}{t} \int_{0}^{t} Y_{s} d s \\
& +\liminf _{t \rightarrow \infty} \frac{1}{t} \int_{0}^{t} f\left(\mathrm{I}_{s}\right) d s+N \sigma \lim _{t \rightarrow \infty} \frac{B_{t}}{t} \\
= & \liminf _{t \rightarrow \infty} \frac{1}{t} \int_{0}^{t} f\left(\mathrm{I}_{s}\right) d s
\end{aligned}
$$




$$
\geq \liminf _{t \rightarrow \infty} \frac{1}{t} \int_{0}^{T} f\left(\mathrm{I}_{s}\right) d s+f\left(x^{*}-\varepsilon\right) \liminf _{t \rightarrow \infty} \frac{t-T}{t}
$$

and hence

$$
\liminf _{t \rightarrow \infty} \frac{1}{t} \ln \left(\frac{\mathrm{I}_{t}}{N-\mathrm{I}_{t}}\right) \geq f\left(x^{*}-\varepsilon\right)>0 .
$$

This gives $\lim _{t \rightarrow \infty} \mathrm{I}_{t}=N$ which contradicts 2.2.5.

To prove the second inequality in 2.2 .4 we proceed as before and assume that there exists $\varepsilon>0$ such that

$$
\mathbb{P}\left(\Omega_{2}\right)>\varepsilon \quad \text { where } \quad \Omega_{2}=\left\{\liminf _{t \rightarrow \infty} I_{t} \geq x^{*}+\varepsilon\right\} .
$$

Therefore, for all $\omega \in \Omega_{2}$, there exists $T(\omega) \geq 0$ such that

$$
\mathrm{I}_{t} \geq x^{*}-\varepsilon, \quad \text { for all } t \geq T(\omega) .
$$

The monotonicity of $f$ gives

$$
f\left(\mathrm{I}_{t}\right) \leq f\left(x^{*}-\varepsilon\right)<0, \quad \text { for all } t \geq T(\omega),
$$

and

$$
\limsup _{t \rightarrow \infty} \frac{1}{t} \ln \left(\frac{\mathrm{I}_{t}}{N-\mathrm{I}_{t}}\right) \leq \limsup _{t \rightarrow \infty} \frac{1}{t} \int_{0}^{t} f\left(\mathrm{I}_{s}\right) d s \leq f\left(x^{*}+\varepsilon\right)<0 .
$$

This implies $\lim _{t \rightarrow \infty} \mathrm{I}_{t}=0$, contradicting 2.2.7.

\subsubsection{Trajectory simulations}

In this section we present various simulations; We consider two different values of $\sigma$ to emphasize that, according to our theoretical results, the limiting behaviour of the solution doesn't depend on them. We also illustrate that the perturbation of $\beta$ proposed here, i.e. via a mean-reverting Ornstein-Uhlenbeck process (whose variance is bounded), differs significantly at level of trajectories of It from the case with Brownian perturbation investigated in [2].

For the first example, set $N=200, i_{0}=100, \beta=0.06, \gamma+\mu=14, \alpha=0.4$, so that $R_{0}^{D}=0.8$ and $\nu=-2$. According to Theorem 2.2 .2 the infection should extinct almost surely. See Figure 2.2.

Next, the same simulation is performed with a different set of parameters. Take $N=200, i_{0}=100, \beta=0.06, \gamma+\mu=12, \alpha=0.4$ which gives $R_{0}^{D}=1$ and $\nu=0$. According to Theorem 2.2.2 the infection should extinct almost surely. See Figure 2.3. 


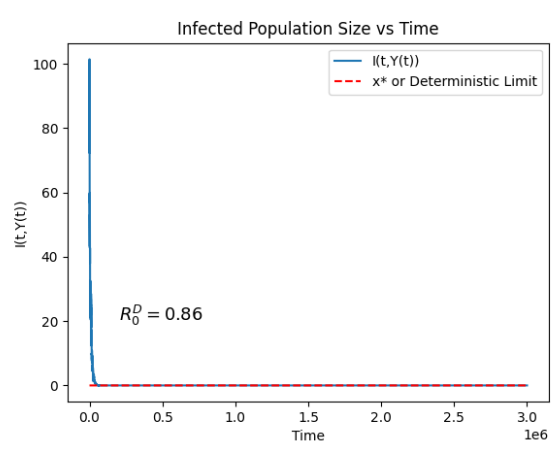

(a) $\sigma=0.005$

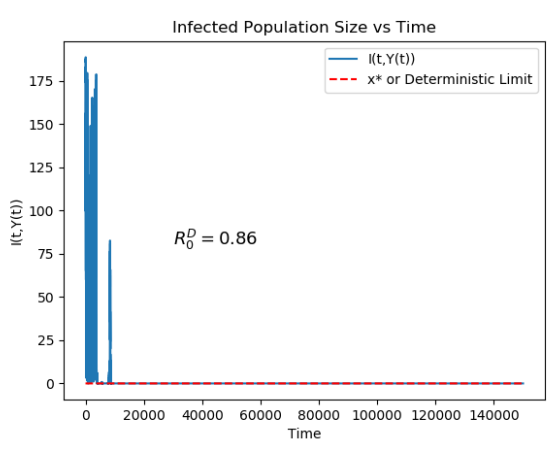

(b) $\sigma=0.05$

Figure 2.2: The plot with parameters $N=200, i_{0}=100, \alpha=0.4, R_{0}^{D}=0.857$ and hence $\nu=-2$. The label for $\mathrm{y}$-axis $I\left(t, Y_{t}\right)$ stands for $\mathrm{I}_{t}$.

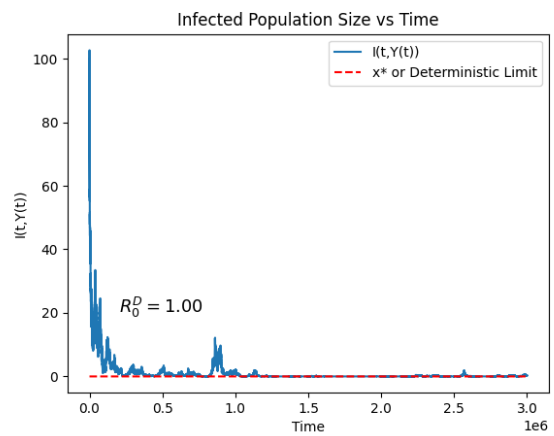

(a) $\sigma=0.005$

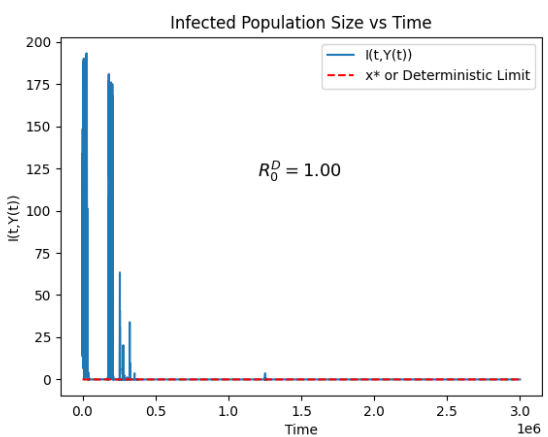

(b) $\sigma=0.05$

Figure 2.3: The plot with parameters $N=200, i_{0}=100, \alpha=0.4, R_{0}^{D}=1$ and hence $\nu=0$. The label for $\mathrm{y}$-axis $I\left(t, Y_{t}\right)$ stands for $\mathrm{I}_{t}$.

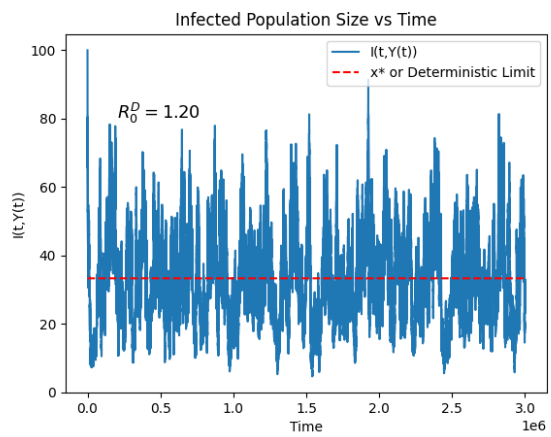

(a) $\sigma=0.005$

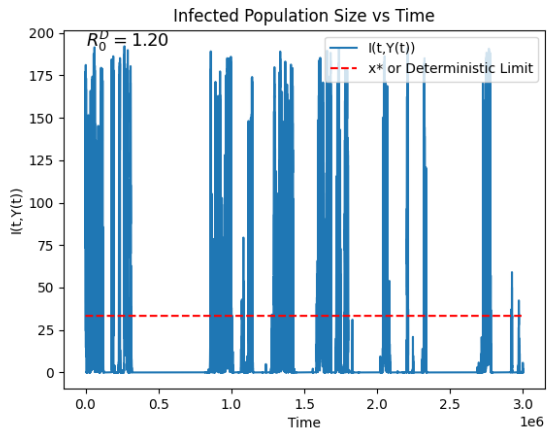

(b) $\sigma=0.05$

Figure 2.4: The plot with parameters $N=200, i_{0}=100, \alpha=0.4$ and $R_{0}^{D}=1.2$ so $\nu=2$. The deterministic limit is 33. The label for y-axis $I\left(t, Y_{t}\right)$ stands for $\mathrm{I}_{t}$. 


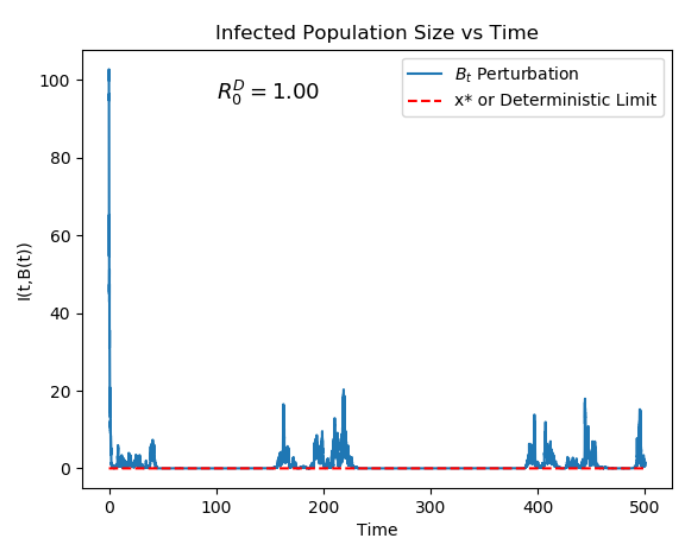

(a) $B_{t}$ Perturbation, $R_{0}^{D}=1.00$

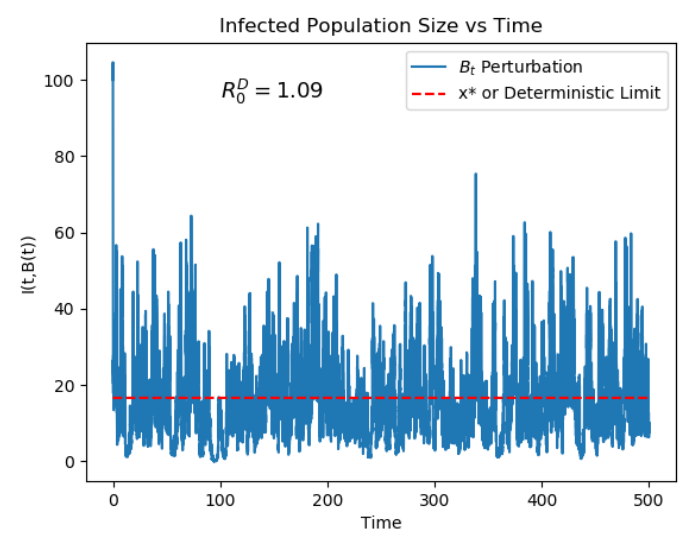

(c) $B_{t}$ Perturbation, $R_{0}^{D}=1.09$

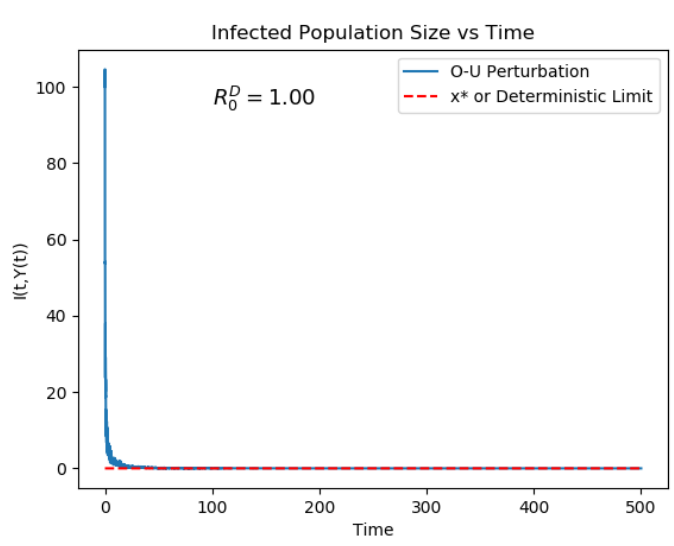

(b) O-U Perturbation, $R_{0}^{D}=1.00$

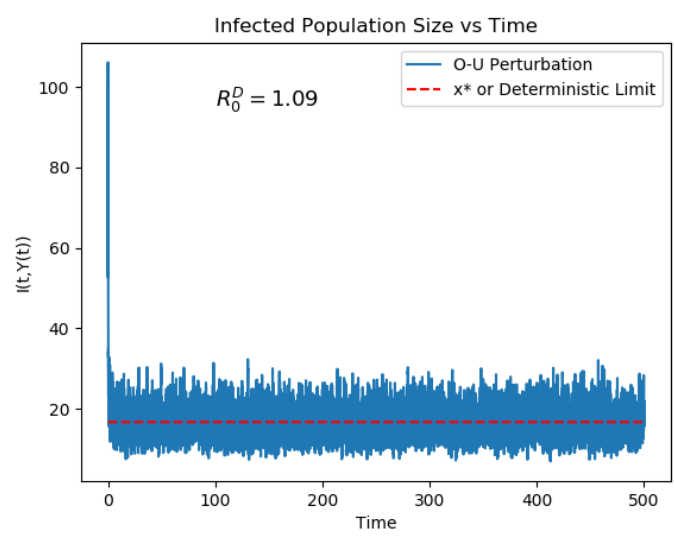

(d) O-U Perturbation, $R_{0}^{D}=1.09$

Figure 2.5: The plot with parameters $N=200, i 0=100, \alpha=10$ and $\sigma=0.005$. The labels for $\mathrm{y}$-axis are $I\left(t, B_{t}\right)$, standing for $\beta$ parameter perturbed with $B_{t}$, and $I\left(t, Y_{t}\right)$, standing for $I_{t}$.

Lastly, consider $N=200, i_{0}=100, \beta=0.06, \gamma+\mu=10, \alpha=0.4$ so $R_{0}^{D}=1.2$ and $\nu=2$. According to Theorem 2.2.2 the infection should persist a.s., or more precisely it should oscillate above and below the deterministic limit. See Figure 2.4 where fluctuations above and below the deterministic limit $x^{*}$ is visible as well.

Lastly, Fig. 2.5 shows a comparison between the trajectories of $I_{t}$ in the presence of Brownian and mean reverting Orstein- Uhlenbeck perturbations of $\beta$ : the boundedness in time of the variance of $Y_{t}$ is clearly observed in the size of the fluctuations of $\mathrm{I}_{t}$. 


\section{Chapter 3}

\section{Generalization of The Parameter Perturbation Process}

\subsection{Stochastic parameter perturbation with a gen- eral process}

In this section we try to understand to which extent the results of the previous sections are determined by the choice of the particular perturbation $\left\{Y_{t}\right\}_{t \geq 0}$ in (2.1.1). To this aim we consider $\left\{Z_{t}\right\}_{t \geq 0}$, solution of the stochastic differential equation

$$
d Z_{t}=b\left(t, Z_{t}\right) d t+\sigma d B_{t}, \quad Z_{0}=0
$$

where $\sigma$ is positive real number and the function $b:[0, T] \times \mathbb{R}$ is assumed to be globally Lipschitz continuous in $z$, uniformly in $t$; We also introduce the corresponding perturbation of the parameter $\beta$, namely

$$
\int_{0}^{t} \beta(s) d s \mapsto \int_{0}^{t}\left(\beta+\frac{d Z_{s}}{d s}\right) d s=\beta t+Z_{t}
$$

If we employ such transformation in (2.0.1), which is the solution of the deterministic SIS model with a time dependent transmission coefficient $\beta$, we get the stochastic process

$$
\mathcal{I}_{t}:=\frac{i_{0} e^{\nu t+N Z_{t}}}{1+\frac{i_{0}}{N}\left(e^{\nu t+N Z_{t}}-1+\int_{0}^{t} e^{\nu s+N Z_{s}}(\gamma+\mu) d s\right)}, \quad t \geq 0
$$

where to ease the notation set $\mathcal{I}_{t}$ instead of $I\left(t, Z_{t}\right)$. Moreover, an application of the Itô formula yields

$$
\begin{aligned}
d \mathcal{I}_{t}= & {\left[\mathcal{I}_{t}\left(N-\mathcal{I}_{t}\right)\left(\frac{\nu}{N}+b\left(t, Z_{t}\right)+\frac{\sigma^{2} N}{2}-\sigma^{2} \mathcal{I}_{t}\right)-\frac{\gamma+\mu}{N} \mathcal{I}_{t}^{2}\right] d t } \\
& +\sigma \mathcal{I}_{t}\left(N-\mathcal{I}_{t}\right) d B_{t}
\end{aligned}
$$


Remark 3.1.1. The restriction to constant diffusion coefficients imposed in (3.1.1) is due the necessity of cross validating the model (3.1.3) also from a differential equations' point of view. In fact, if one smooths the process $\left\{Z_{t}\right\}_{t \geq 0}$ as

$$
\frac{d Z_{t}^{\pi}}{d t}=b\left(t, Z_{t}^{\pi}\right)+\sigma \frac{d B_{t}^{\pi}}{d t}, \quad Z_{0}^{\pi}=0
$$

and perturb correspondingly the parameter $\beta$ in equation 1.2 .2 , we obtain

$$
\begin{aligned}
\frac{d \mathcal{I}_{t}^{\pi}}{d t}= & \beta \mathcal{I}_{t}^{\pi}\left(N-\mathcal{I}_{t}^{\pi}\right)-(\mu+\gamma) \mathcal{I}_{t}^{\pi}+b\left(t, Z_{t}^{\pi}\right) \mathcal{I}_{t}^{\pi}\left(N-\mathcal{I}_{t}^{\pi}\right) \\
& +\sigma \mathcal{I}_{t}^{\pi}\left(N-\mathcal{I}_{t}^{\pi}\right) \frac{d B_{t}^{\pi}}{d t} .
\end{aligned}
$$

According to the Wong-Zakai Theorem the unique solution $\left\{\mathcal{I}_{t}^{\pi}\right\}_{t \in[0, T]}$ of the random ordinary differential equation above converges, as the mesh of the partition $\pi$ tends to zero, to the solution of the Stratonovich stochastic differential equation

$$
d \mathcal{I}_{t}=\left[\beta \mathcal{I}_{t}\left(N-\mathcal{I}_{t}\right)-(\mu+\gamma) \mathcal{I}_{t}+b\left(t, Z_{t}\right) \mathcal{I}_{t}\left(N-\mathcal{I}_{t}\right)\right] d t+\sigma \mathcal{I}_{t}\left(N-\mathcal{I}_{t}\right) \circ d B_{t},
$$

which in turn is equivalent to the Itô SDE

$$
\begin{aligned}
d \mathcal{I}_{t}= & {\left[\beta \mathcal{I}_{t}\left(N-\mathcal{I}_{t}\right)-(\mu+\gamma) \mathcal{I}_{t}+b\left(t, Z_{t}\right) \mathcal{I}_{t}\left(N-\mathcal{I}_{t}\right)+\frac{\sigma^{2}}{2} \mathcal{I}(t)(N-\mathcal{I}(t))(N-2 \mathcal{I}(t))\right] d t } \\
& +\sigma \mathcal{I}_{t}\left(N-\mathcal{I}_{t}\right) d B_{t} .
\end{aligned}
$$

This SDE coincides with (3.1.4) thus validating that model. If we allow $\sigma$ in (3.1.1) to depend also on $Z$, then this match wouldn't take place for the presence of an additional drift term in the equation for $\left\{Z_{t}\right\}_{t \geq 0}$.

We now start to analyse the properties of $\left\{\mathcal{I}_{t}\right\}_{t \geq 0}$ by stating the analogue of Proposition 2.2.1.

Proposition 3.1.2. For the stochastic process $\left\{\mathcal{I}_{t}\right\}_{t \geq 0}$ defined in (3.1.3) we have

$$
\mathbb{P}\left(\mathcal{I}_{t} \in\right] 0, N[)=1, \quad \text { for all } t \geq 0 .
$$

Proof. Looking through the proof of Proposition 2.2.1 one easily see that the same conclusion holds for $\left\{\mathcal{I}_{t}\right\}_{t \geq 0}$.

The next theorem provides a sufficient condition on the stochastic process $\left\{Z_{t}\right\}_{t \geq 0}$ which guarantees extinction for $\left\{\mathcal{I}_{t}\right\}_{t \geq 0}$.

Theorem 3.1.3. Assume that $R_{0}^{D}=\frac{\beta N}{\gamma+\mu} \leq 1$, or equivalently $\nu=\beta N-(\gamma+\nu) \leq 0$. If

$$
\limsup _{t \rightarrow \infty} \frac{Z_{t}}{t} \leq 0, \quad \text { almost surely }
$$

then

$$
\lim _{t \rightarrow \infty} \mathcal{I}_{t}=0 \quad \text { almost surely. }
$$


Proof. Take $G(y):=\ln \left(\frac{y}{N-y}\right)$, for $\left.y \in\right] 0, N[$; an application of the Itô formula gives

$$
d G\left(\mathcal{I}_{t}\right)=\left[\nu-(\gamma+\mu) \frac{\mathcal{I}_{t}}{N-\mathcal{I}_{t}}\right] d t+N d Z_{t}
$$

this yields

$$
G\left(\mathcal{I}_{t}\right)=\ln \left(\frac{i_{0}}{N-i_{0}}\right)+\int_{0}^{t} f\left(\mathcal{I}_{s}\right) d s+N Z_{t},
$$

where $f$ is defined as $(2.2 .2)$ again. By utilizing the monotonicity of $f$, we get

$$
\limsup _{t \rightarrow \infty} \frac{1}{t} G\left(\mathcal{I}_{t}\right)<\nu+N \limsup _{t \rightarrow \infty} \frac{Z_{t}}{t} .
$$

Now, if

$$
\limsup _{t \rightarrow \infty} \frac{Z_{t}}{t} \leq 0, \quad \text { almost surely }
$$

then $\nu \leq 0$ implies immediately that

$$
\limsup _{t \rightarrow \infty} \frac{1}{t} \ln \left(\frac{\mathcal{I}_{t}}{N-\mathcal{I}_{t}}\right)<0
$$

The last inequality entails the statement of our theorem and competes the proof.

Now the analogue of Theorem 2.2 .3 will be stated.

Theorem 3.1.4. Assume that $R_{0}^{D}>1$, or equivalently $\nu>0$. If

$$
0 \leq \liminf _{t \rightarrow \infty} \frac{Z_{t}}{t} \leq \limsup _{t \rightarrow \infty} \frac{Z_{t}}{t}<+\infty
$$

then the infection is persistent. More precisely, for all $x \in] 0, N[$ we have

$$
\liminf _{t \rightarrow \infty} \mathcal{I}_{t} \leq x \leq \limsup _{t \rightarrow \infty} \mathcal{I}_{t}, \quad \text { almost surely. }
$$

Proof. We now proceed by contradiction as in the proof of Theorem 2.2.3. Similarly, take $G(y):=\ln \left(\frac{y}{N-y}\right)$, for $\left.y \in\right] 0, N[$; an application of the Itô formula gives

$$
d G\left(\mathcal{I}_{t}\right)=\left[\nu-(\gamma+\mu) \frac{\mathcal{I}_{t}}{N-\mathcal{I}_{t}}\right] d t+N d Z_{t}
$$

Now fix $0<x<N$ and first prove that

$$
\limsup _{t \rightarrow \infty} \mathcal{I}_{t} \geq x, \quad \text { almost surely. }
$$


Assume to the contrary that the event $\left\{\lim \sup _{t \rightarrow \infty} \mathcal{I}_{t}<x\right\}$ has positive probability. This means that there exists $T(\omega) \geq 0$ such that $\mathcal{I}_{t}<x$, for all $t \geq T(\omega)$; this, together with the monotonicity of $(2.2 .2)$, gives

$$
\begin{aligned}
\liminf _{t \rightarrow \infty} \frac{1}{t} \ln \left(\frac{\mathcal{I}_{t}}{N-\mathcal{I}_{t}}\right) & \geq \liminf _{t \rightarrow \infty} \frac{1}{t} \int_{0}^{t} f\left(\mathcal{I}_{s}\right) d s+N \liminf _{t \rightarrow \infty} \frac{Z_{t}}{t} \\
& >f(x)+N \liminf _{t \rightarrow \infty} \frac{Z_{t}}{t} .
\end{aligned}
$$

Now, the core of Theorem 2.2.3 is the left hand side above being positive and this is guaranteed if

$$
N \liminf _{t \rightarrow \infty} \frac{Z_{t}}{t} \geq-f(x)
$$

In this case $\liminf \operatorname{in}_{t \rightarrow \infty} \frac{1}{t} \ln \left(\frac{\mathcal{I}_{t}}{N-\mathcal{I}_{t}}\right)>0$ and hence $\lim _{t \rightarrow \infty} \mathcal{I}_{t}=N$, contradicting our initial assumption. Since the range of $f$ is $]-\infty, \nu]$, condition $(3.1 .8)$ is implied by the first inequality in (3.1.6) and positivity of $\nu$.

We now prove that

$$
\liminf _{t \rightarrow \infty} \mathcal{I}_{t} \leq x, \quad \text { almost surely. }
$$

Assume to the contrary that the event $\left\{\lim _{\inf } \lim _{t \rightarrow \infty} \mathcal{I}_{t}>x\right\}$ has positive probability; this implies the existence of $T(\omega)>0$ such that $\mathcal{I}_{t}>x$, for all $t \geq T(\omega)$. Then,

$$
\begin{aligned}
\limsup _{t \rightarrow \infty} \frac{1}{t} \ln \left(\frac{\mathcal{I}_{t}}{N-\mathcal{I}_{t}}\right) & \leq \limsup _{t \rightarrow \infty} \int_{0}^{t} f\left(\mathcal{I}_{s}\right) d s+N \limsup _{t \rightarrow \infty} \frac{Z_{t}}{t} \\
& <f(x)+N \limsup _{t \rightarrow \infty} \frac{Z_{t}}{t} .
\end{aligned}
$$

Now, the core of Theorem 2.2.3 is the left hand side above being negative and this is guaranteed if

$$
N \limsup _{t \rightarrow \infty} \frac{Z_{t}}{t} \leq-f(x)
$$

In this case $\lim \sup _{t \rightarrow \infty} \frac{1}{t} \ln \left(\frac{\mathcal{I}_{t}}{N-\mathcal{I}_{t}}\right)<0$ and hence $\lim _{t \rightarrow \infty} \mathcal{I}_{t}=0$ which contradicts our initial assumption. Since the range of $f$ is $]-\infty, \nu]$, condition $(3.1 .9)$ is implied by the last inequality in 3.1.6.

Remark 3.1.5. If the process $\left\{Z_{t}\right\}_{t \geq 0}$ satisfies both 3.1.5) and (3.1.6), then for $\nu \leq 0$ one has extinction and for $\nu>0$ one has persistence for the associated model. It is useful to note this is the case only when

$$
\lim _{t \rightarrow \infty} \frac{Z_{t}}{t}=0
$$




\subsection{Trajectory simulations}

In this section a simple example will be given to support the theoretical results of Theorems 3.1.3 and 3.1.4. We choose

$$
Z_{t}:=\alpha t+\sigma B_{t}
$$

and note that

$$
\limsup _{t \rightarrow \infty} \frac{Z_{t}}{t}=\liminf _{t \rightarrow \infty} \frac{Z_{t}}{t}=\alpha, \quad \text { almost surely. }
$$

If $\alpha<0$, then the assumption of Theorem 3.1 .3 is fulfilled while those of Theorem 3.1 .4 are not. This means that for $R_{0}^{D} \leq 1$, or equivalently $\nu \leq 0$, the extinction is guaranteed; however, for $R_{0}^{D}>1$, or equivalently $\nu>0$, the persistence of infection is not guaranteed.

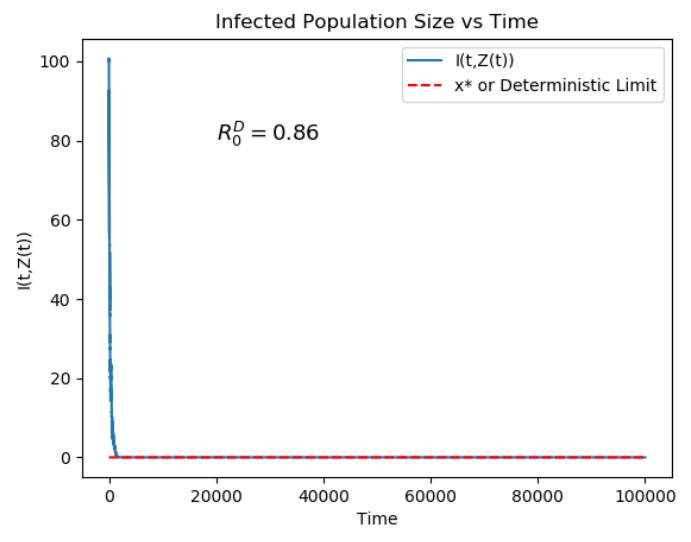

(a) $R_{0}^{D}=0.857$

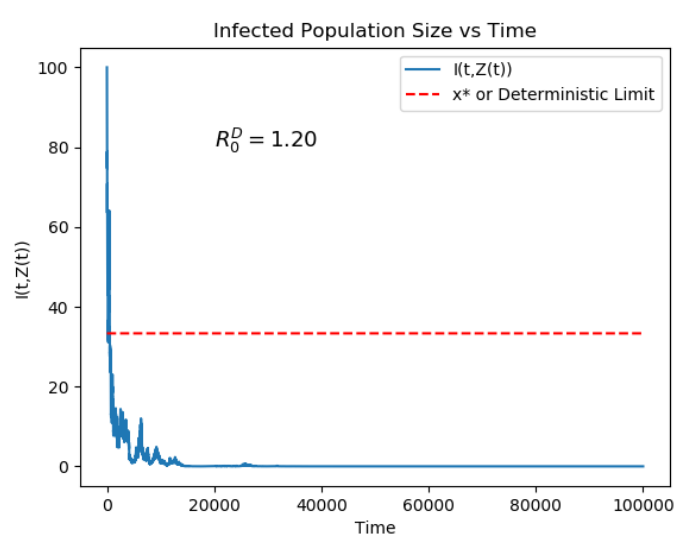

(c) $R_{0}^{D}=1.200$

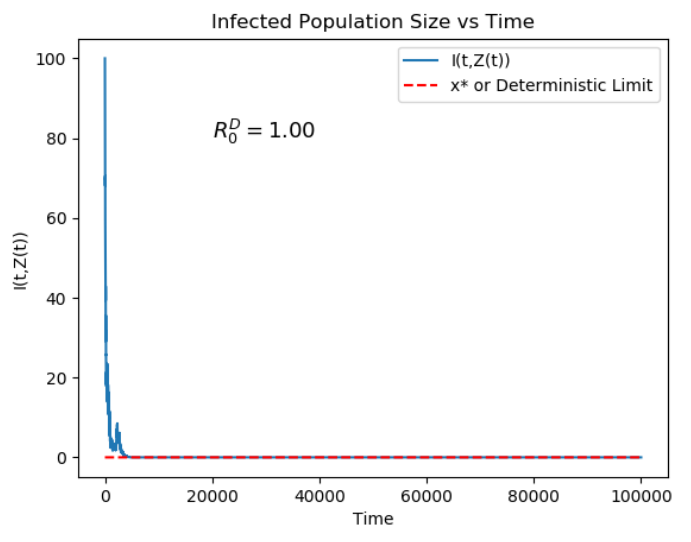

(b) $R_{0}^{D}=1.000$

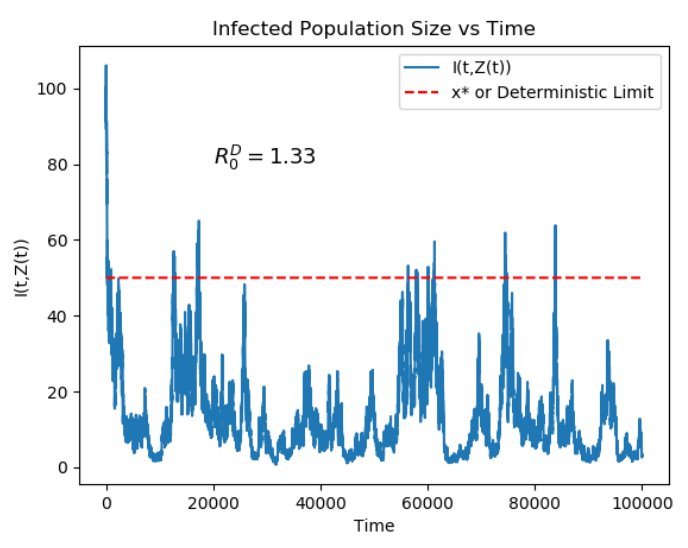

(d) $R_{0}^{D}=1.333$

Figure 3.1: The plot with parameters $N=200, i_{0}=100, \alpha=-0.011$ and $\sigma=0.005$. The label for y-axis $I\left(t, Z_{t}\right)$ stands for $\mathcal{I}_{t}$. 
Figure 3.1 supports this claim. As one can see the panels $3.1 \mathrm{a}$ and $3.1 \mathrm{~b}$ shows the extinction of the infection since Theorem 3.1 .3 is satisfied. However, panels $3.1 \mathrm{c}$ and $3.1 \mathrm{~d}$ shows both the examples of extinction and persistence even though the $\nu>0\left(R_{0}^{D}>1\right)$. Thus one looses the properties of the deterministic model.

A complementary analysis can be made for $\alpha>0$. In this case the assumption of Theorem 3.1.4 will be satisfied while those of Theorem 3.1.3 are not. This indicates that the infection will be persistent as long as $R_{0}^{D}>1$; however, the extinction of infection is not guaranteed for $R_{0}^{D} \leq 1$. The results of Figure 3.2 support this claim.

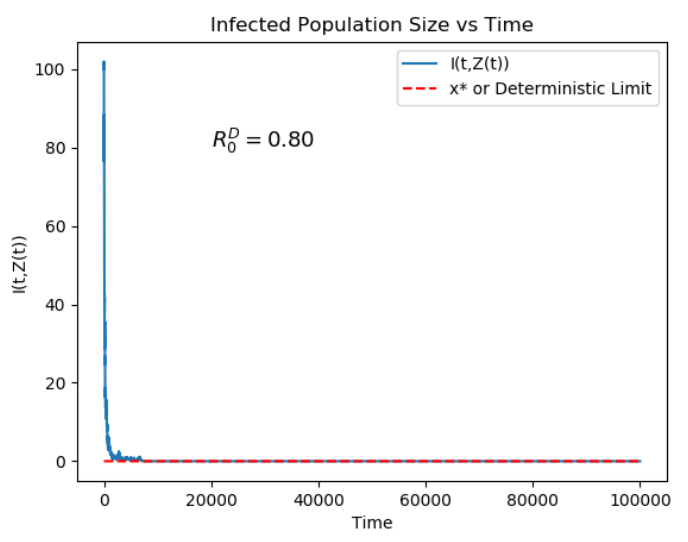

(a) $R_{0}^{D}=0.800$

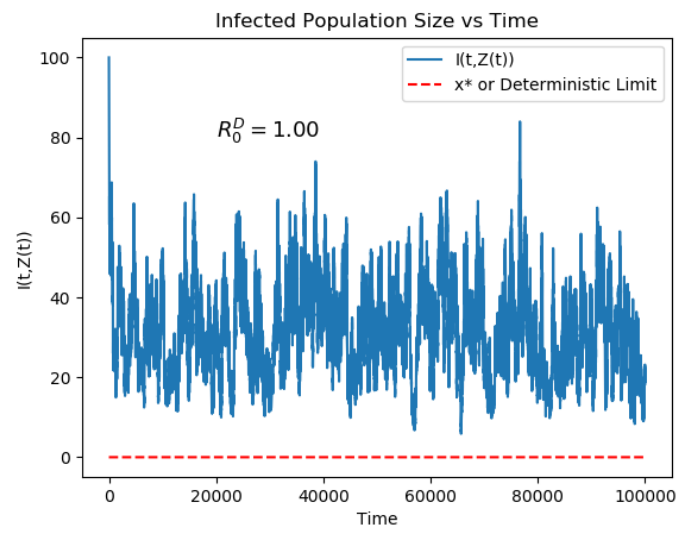

(c) $R_{0}^{D}=1.000$

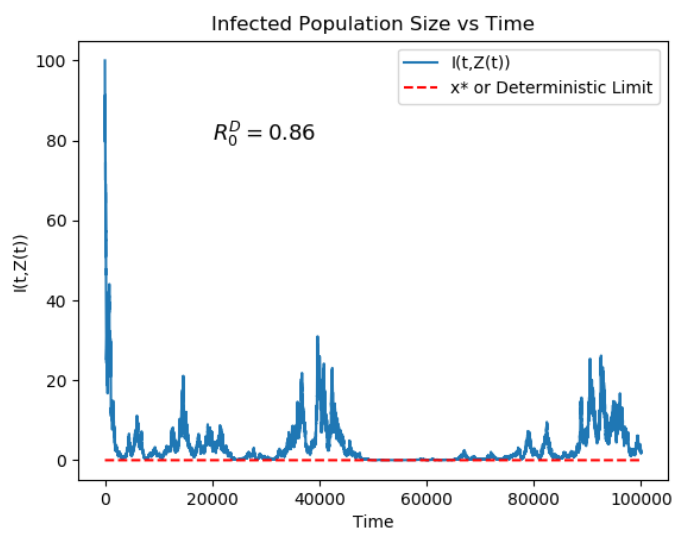

(b) $R_{0}^{D}=0.857$

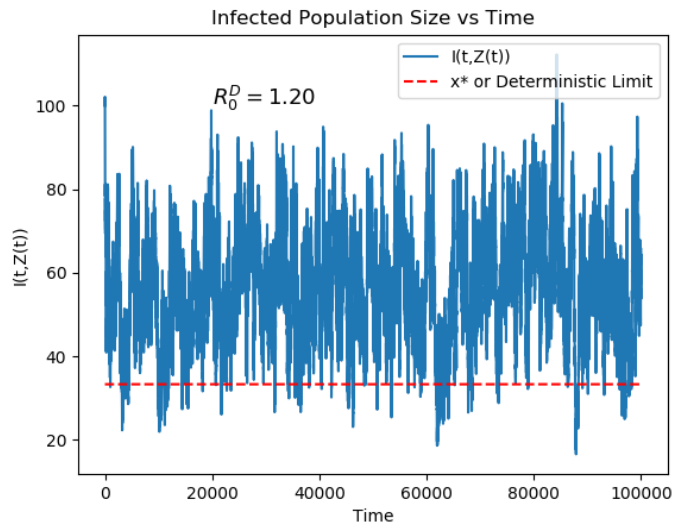

(d) $R_{0}^{D}=1.200$

Figure 3.2: The plot with parameters $N=200, i_{0}=100, \alpha=-0.011$ and $\sigma=0.005$. The label for y-axis $I\left(t, Z_{t}\right)$ stands for $\mathcal{I}_{t}$.

In the panel $3.2 \mathrm{~d}$, the infection is persistent as expected. However, the extinction is not seen in panels $3.2 \mathrm{c}$ and $3.2 \mathrm{~b}$ since the sufficient condition in Theorem 3.1 .3 is not satisfied. Because the condition was sufficient and not necessary, although it is not satisfied, the extinction can still happen for $R_{0}^{D} \leq 1$, as shown in panel 3.2a.

One last important comment is that, when $\alpha=0$, then one has the same kind of 
perturbation of the $\beta$ parameter utilized in [5], so now $Z_{t}=\sigma B_{t}$. If the $\beta$ parameter is perturbed in the same way as this study and not in [5], since $\lim _{t \rightarrow \infty} Z_{t} / t=0$, by the remark 3.1.5 it can be said that, for $R_{0}^{D} \leq 1(\nu \leq 0)$ cases there will be extinction and for cases $R_{0}^{D}>1(\nu>0)$ there will be persistence of infection. We would like to stress that conditions are not the same as published in [5] because the perturbation method is different in this study. 


\section{Chapter 4}

\section{Conclusion and Discussion}

In this thesis, the basic background necessary to follow the analysis is provided in Chapter 1. Later in Chapter 2 we propose a new perturbation method for the disease transmission coefficient in SIS model. Our approach consists in acting directly on the explicit solution of the deterministic problem, thus avoiding delicate manipulations of differential quantities such as $d B_{t}$. Nevertheless, our model is cross validated at the level of differential equations once we smooth the perturbation and use the Wong-Zakai theorem. We first use this method with a perturbation of mean reverting type and properties of the corresponding model are analysed. Then, generalization to different sources of randomness are investigated.

When the deterministic SIS model $(1.2 .1)$ is perturbed with a mean reverting OrnsteinUhlenbeck process $\left\{Y_{t}\right\}_{t \geq 0}$, the solution $\left\{\mathrm{I}_{t}\right\}_{t \geq 0}$ is shown to preserve the deterministic model's regimes for extinction and persistence similar to expression (1.2.4). Namely:

- if $R_{0}^{D} \leq 1$, then we have extinction of infection;

- if $R_{0}^{D}>1$, then we have persistence of infection.

Then, in Chapter 3 some simple sufficient conditions on the class of possible perturbations which entail the same key feature for the corresponding models were identified. It turns out that if a general stochastic process $Z_{t}$, solving the general SDE (3.1.1), satisfies both Theorems 3.1.3 and 3.1.4, then the perturbed SIS Model with $Z_{t}$ perturbation in the $\beta$ parameter, behaves similar to its deterministic version. That is:

- if $R_{0}^{D} \leq 1$, then we have extinction of infection;

- if $R_{0}^{D}>1$, then we have persistence of infection.

That is a very useful result for other researchers to use. This way, if anyone wants to perturb a SIS model like system of ODEs, they can use the same perturbation method and a $Z_{t}$ process so that it is known that the solution exists, it is strong and unique, lies between $] 0, N[$ and the limiting behaviour is similar to the original model.

This study emphasizes that the methodology of perturbation of deterministic models is crucial in generating different stochastic versions. For further studies one can apply 
the same rationale to other parameters in the deterministic SIS model, i.e. $\gamma+\mu$, or to other models, such as Lotka-Volterra type of equations ([9],[12]). 


\section{Bibliography}

[1] Allen, E., Modelling with Itô Stochastic Differential Equations, Springer-Verlag, 2007.

[2] E. Bernardi and A.Lanconelli, A note about the invariance of the basic reproduction number for stochastically perturbed SIS models, arXiv:2005.04973v2.

[3] F. Brauer, L.J.S. Allen, P. Van den Driessche and J. Wu, Mathematical Epidemiology, Lecture Notes in Mathematics, No. 1945, Mathematical Biosciences Subseries, 2008.

[4] Cai, Y., Jiao, J., Gui, Z., Liu, Y., \& Wang, W. (2018). Environmental variability in a stochastic epidemic model. Applied Mathematics and Computation, 329, 210-226. doi:10.1016/j.amc.2018.02.009

[5] A. Gray, D. Greenhalgh, L. Hu, X. Mao, J. Pan, A stochastic differential equation SIS epidemic model, SIAM J. Appl. Math. 71 (3) (2011) 876-902.

[6] Hethcote, H.W. and Yorke, J.A., Gonorrhea Transmission Dynamics and Control, Lecture Notes in Biomathematics 56, Springer-Verlag, 1994.

[7] Jin, Y., Wang, W., \& Xiao, S. (2007). An sirs model with a nonlinear incidence rate. Chaos, SolItôns \& Fractals, 34(5), 1482-1497. doi:10.1016/j.chaos.2006.04.022

[8] I. Karatzas and S. E. Shreve, Brownian Motion and stochastic calculus, SpringerVerlag, New York, 1991.

[9] A. J. Lotka, Contribution to quantitative parasitology, J. Wash. Acad. Sci. 13 (1923) 152-158.

[10] Mao, X. (2011). Stochastic differential equations and applications. In Stochastic differential equations and applications (Second ed., pp. 12-13). Oxford: Woodhead.

[11] D. W. Stroock and S. R. S. Varadhan, On the support of diffusion processes with applications to the strong maximum principle, Proceedings 6-th Berkeley Symposium Math. Statist. Probab. 3 (1972) University of California Press, Berkeley, 333-359.

[12] V. Volterra, Variazioni e fluttuazioni del numero d'individui in specie animali conviventi. Mem. Acad. Lincei 2 (1926) 31-113. 
[13] E. Wong and M. Zakai: On the relation between ordinary and stochastic differential equations, Intern. J. Engr. Sci. 3 (1965) 213-229.

[14] Xu, C. (2017). Global threshold dynamics of a stochastic differential equation sis model. Journal of Mathematical Analysis and Applications, 447(2), 736-757. doi:10.1016/j.jmaa.2016.10.041

[15] Zhou, Y., \& Liu, H. (2003). Stability of periodic solutions for an Sis model with Pulse vaccination. Mathematical and Computer Modelling, 38(3-4), 299-308. doi:10.1016/s0895-7177(03)90088-4

[16] Kuo, H. (2006). Introduction to stochastic integration. New York: Springer.

[17] Lanconelli, A., \& Perçin, B. T. (2022). On a new method for the Stochastic perturbation of the disease transmission coefficient IN SIS models. Applied Mathematics and Computation, 413, 126600. https://doi.org/10.1016/j.amc.2021.126600

[18] Resnick, S. I. (2014). A probability path. Birkhäuser. 